DO-TH 07/06

August 2007

\title{
Dynamical parton distributions of the nucleon and very small-x physics
}

\author{
M. Glück, P. Jimenez-Delgado, E. Reya \\ Universität Dortmund, Institut für Physik \\ D-44221 Dortmund, Germany
}

\begin{abstract}
Utilizing recent DIS measurements $\left(F_{2, \mathrm{~L}}\right)$ and data on dilepton and high- $E_{\mathrm{T}}$ jet production we determine the dynamical parton distributions of the nucleon generated radiatively from valence-like positive input distributions at optimally chosen low resolution scales. These are compared with 'standard' distributions generated from positive input distributions at some fixed and higher resolution scale. It is shown that up to the next to leading order $\mathrm{NLO}(\overline{\mathrm{MS}}$, DIS $)$ of perturbative QCD considered in this paper, the uncertainties of the dynamical distributions are, as expected, smaller than those of their standard counterparts. This holds true in particular in the presently unexplored extremely small $-x$ region relevant for evaluating ultrahigh energy cross sections in astrophysical applications. It is noted that our new dynamical distributions are compatible, within the presently determined uncertainties, with previously determined dynamical parton distributions.
\end{abstract}




\section{Introduction}

The parton distributions of the nucleon are extracted from deep inelastic scattering data by essentially two different approaches which differ in their choice of the input distributions at some low scale $Q_{0}$. In the common approach, e.g. [1, 2, 3], $Q_{0}$ is fixed at some arbitrarily chosen $Q_{0}>1 \mathrm{GeV}$ and the corresponding input distributions are unrestricted, allowing even for negative gluon distributions [3, 4, 5] in the small Bjorken- $x$ region, i.e. negative cross sections like $F_{\mathrm{L}}\left(x, Q^{2}\right)$. Alternatively [6, 7, 8] the parton distributions at $Q \gtrsim 1$ $\mathrm{GeV}$ are QCD radiatively generated from valence-like positive input distributions at an optimally determined $Q_{0} \equiv \mu<1 \mathrm{GeV}$ (where 'valence-like' refers to $a_{f}>0$ for all input distributions $\left.x f\left(x, \mu^{2}\right) \propto x^{a_{f}}(1-x)^{b_{f}}\right)$. This more restrictive ansatz implies, of course, less uncertainties concerning the behavior of the parton distributions in the small $-x$ region at $Q>\mu$ which is entirely due to QCD dynamics. In particular it provided unique (steep) predictions [6, 9] for the experimentally unexplored region $x<10^{-2}$ which were subsequently first confirmed in [10, 11].

This predictive power is especially important for investigations concerning cross sections [12] of ultrahigh energy particles (neutrinos) produced via astrophysical acceleration processes, e.g. in active galactic nuclei, black holes or in the decays of very massive particles (see, for example, [13, 14, 15]). Here one needs a somewhat reliable knowledge of parton distributions at the weak scale $Q^{2}=M_{W}^{2}$ down to $x \simeq 10^{-9}\left(x \simeq M_{W}^{2} / 2 m_{N} E_{\nu}\right)$ at highest energies of $E_{\nu} \simeq 10^{12} \mathrm{GeV}$ which requires extrapolations into the yet unmeasured small- $x$ region $x<10^{-3}$. Furthermore this 'radiative' approach based on QCD dynamics is also useful for connecting nonperturbative models valid at $Q<1 \mathrm{GeV}$ (like chiral quark-soliton models [16-20] and statistical parton models [21-24]) with the actually measured distributions at $Q>1 \mathrm{GeV}$.

In the present paper we confront recent precision deep inelastic scattering (DIS) ep data, Drell-Yan dilepton and high $-p_{\mathrm{T}}$ jet data with radiatively generated parton distri- 
butions arising from a valence-like positive input at $Q<1 \mathrm{GeV}$, following and extending the latest GRV98 analysis [8]. Moreover we study the dependence and stability of the small- $x$ predictions, in particular of their extrapolations down to $10^{-9}$, with respect to a different choice of the factorization scheme ( $\overline{\mathrm{MS}}$ versus DIS). Furthermore we compare these 'dynamical' results with the ones obtained from the common evolution approach being based on a non-valence-like input at $Q_{0}>1 \mathrm{GeV}$. In particular we shall compare their associated uncertainties. As should be clear by now, it will turn out that these uncertainties are indeed smaller for the radiatively generated parton distributions, particularly in the small- $x$ region, due to their valence-like input and the sizeably larger evolution distance starting at $Q_{0}<1 \mathrm{GeV}$.

\section{Formalism}

The aforementioned analyses are undertaken at the next-to-leading order (NLO) of perturbative QCD within the modified minimal substraction $(\overline{\mathrm{MS}})$ factorization and renormalization scheme. For the radiative model we shall also present results as obtained within the so-called DIS factorization scheme [25, 26]. Heavy quarks $(c, b, t)$ will not be considered as partons, i.e. the number of active flavors $n_{f}$ appearing in the splitting functions and the corresponding Wilson coefficients will be fixed, $n_{f}=3$. This defines the so-called 'fixed-flavor number scheme' (FFNS). As argued in [27], it is nevertheless consistent and correct to utilize the standard variable $n_{f}$ scheme for the $\beta$-function, and we shall adopt this procedure in our evaluation of the running coupling constant $\alpha_{s}\left(Q^{2}\right)$. Up to NLO, the strong coupling $a\left(Q^{2}\right) \equiv \alpha_{s}\left(Q^{2}\right) / 4 \pi$ evolves according to

$$
d a / d \ln Q^{2}=-\beta_{0} a^{2}-\beta_{1} a^{3}
$$

where $\beta_{0}=11-2 n_{f} / 3$ and $\beta_{1}=102-38 n_{f} / 3$. Here we utilize the exact numerical (iterative) solution for $a\left(Q^{2}\right)$ since it is mandatory in the low $Q^{2}$ region [8] relevant for 
the valence-like approach. The exact solution of (1) can be written implicitly

$$
\ln \frac{Q^{2}}{\tilde{\Lambda}^{2}}=\frac{1}{\beta_{0} a\left(Q^{2}\right)}-\frac{\beta_{1}}{\beta_{0}^{2}} \ln \left(\frac{1}{\beta_{0} a\left(Q^{2}\right)}+\frac{\beta_{1}}{\beta_{0}^{2}}\right) .
$$

Since $\beta_{0,1}$ are not continuous for different $n_{f}$, the continuity of $a\left(Q^{2}\right)$ requires to choose different values for the integration constant $\tilde{\Lambda}$ for different flavor numbers $n_{f}, \tilde{\Lambda}^{\left(n_{f}\right)}$, which are fixed by the $a\left(Q^{2}\right)$ matchings at $Q=m_{c, b, t}$. We have chosen

$$
m_{c}=1.3 \mathrm{GeV}, \quad m_{b}=4.2 \mathrm{GeV}, \quad m_{t}=175 \mathrm{GeV},
$$

which turn out to be the optimal choices for all our subsequent LO and NLO analyses of heavy quark production. This exact solution reduces to the common approximate 'asymptotic' solution

$$
a\left(Q^{2}\right) \simeq \frac{1}{\beta_{0} \ln \left(Q^{2} / \Lambda^{2}\right)}-\frac{\beta_{1}}{\beta_{0}^{3}} \frac{\ln \ln \left(Q^{2} / \Lambda^{2}\right)}{\left[\ln \left(Q^{2} / \Lambda^{2}\right)\right]^{2}}
$$

which turns out to be sufficiently accurate for $Q^{2} \gtrsim 2 \mathrm{GeV}^{2}$ and which, moreover, is easier to use for practical applications of our results. The values for $\Lambda^{\left(n_{f}\right)}$, as well as for $\tilde{\Lambda}^{\left(n_{f}\right)}$, corresponding to our various LO and NLO global dynamical fits are given as follows: In LO, where $\beta_{1} \equiv 0$ and $\tilde{\Lambda}_{\mathrm{LO}}^{\left(n_{f}\right)}=\Lambda_{\mathrm{LO}}^{\left(n_{f}\right)}, \Lambda_{\mathrm{LO}}^{(3,4,5,6)}=210.3,181.8,138.3,70.1 \mathrm{MeV}$; in $\operatorname{NLO}(\overline{\mathrm{MS}}) \tilde{\Lambda}_{\overline{\mathrm{MS}}}^{(3,4,5,6)}=302.8,251.0,172.8,70.0 \mathrm{MeV}$ and $\Lambda_{\overline{\mathrm{MS}}}^{(4,5,6)}=269.7,184.5,72.9 \mathrm{MeV}$; in $\mathrm{NLO}(\mathrm{DIS}) \tilde{\Lambda}_{\mathrm{DIS}}^{(3,4,5,6)}=288.5,238.0,162.9,65.6 \mathrm{MeV}$ while $\Lambda_{\mathrm{DIS}}^{(4,5,6)}=255.9,173.9,68.3$ $\mathrm{MeV}$.

Let us now turn to the update of our LO and $\mathrm{NLO}(\overline{\mathrm{MS}})$ GRV98 distributions [8] which consists of a fine tuning of the valence-like input densities $x f\left(x, Q_{0}^{2}\right)$ as well as of the input scale $Q_{0} \equiv \mu<1 \mathrm{GeV}$. The non-singlet input densities $u_{v}, d_{v}, \Delta \equiv \bar{d}-\bar{u}$ and the valence-like input distributions $\bar{d}+\bar{u}, \bar{s}=s$ and $g$ in the singlet sector are generically parametrized as

$$
x f\left(x, Q_{0}^{2}\right)=N_{f} x^{a_{f}}(1-x)^{b_{f}}\left(1+A_{f} \sqrt{x}+B_{f} x\right)
$$

subject to the constraints $\int_{0}^{1} u_{v} d x=2, \int_{0}^{1} d_{v} d x=1$ and

$$
\int_{0}^{1} x\left[u_{v}+d_{v}+2(\bar{u}+\bar{d}+\bar{s})+g\right] d x=1
$$


Since the data sets we are using are insensitive to the specific choice of the strange quark distributions, we continue to generate the strange densities entirely radiatively [8] starting from $s\left(x, Q_{0}^{2}\right)=\bar{s}\left(x, Q_{0}^{2}\right)=0$ in the valence-like approach where $Q_{0}<1 \mathrm{GeV}$. In the common standard approach where $Q_{0}>1 \mathrm{GeV}$ we choose as usual $s\left(x, Q_{0}^{2}\right)=$ $\bar{s}\left(x, Q_{0}^{2}\right)=\left[\bar{u}\left(x, Q_{0}^{2}\right)+\bar{d}\left(x, Q_{0}^{2}\right)\right] / 4$. Furthermore, since all our fits did not require the additional polynomial in (5) for the gluon distribution, we have set $A_{g}=B_{g}=0$. This left us with a total of 21 independent fit parameters, including $\alpha_{s}$.

These free parameters have been fixed using the following data sets. The HERA ep measurements [28, 29] for $Q^{2} \geq 2 \mathrm{GeV}^{2}$ for the 'reduced' DIS one-photon exchange cross section $\sigma_{r}=F_{2}-\left(y^{2} / Y_{+}\right) F_{\mathrm{L}}$ together with the full neutral current $\left(\gamma, Z^{0}\right)$ sector [30]

$$
\sigma_{r, \mathrm{NC}}^{e^{ \pm} p}\left(x, Q^{2}\right) \equiv\left(\frac{2 \pi \alpha^{2} Y_{+}}{x Q^{4}}\right)^{-1} \frac{d^{2} \sigma_{\mathrm{NC}}^{e^{ \pm} p}}{d x d Q^{2}}=F_{2}^{\mathrm{NC}}-\frac{y^{2}}{Y_{+}} F_{\mathrm{L}}^{\mathrm{NC}} \mp \frac{Y_{-}}{Y_{+}} x F_{3}^{\mathrm{NC}}
$$

where $Y_{ \pm}=1 \pm(1-y)^{2}$ and

$$
\begin{aligned}
& F_{2, \mathrm{~L}}^{\mathrm{NC}}=F_{2, \mathrm{~L}}-v_{e} \kappa F_{2, \mathrm{~L}}^{\gamma Z}+\left(v_{e}^{2}+a_{e}^{2}\right) \kappa^{2} F_{2, \mathrm{~L}}^{Z} \\
& F_{3}^{\mathrm{NC}}=-a_{e} \kappa F_{3}^{\gamma Z}+\left(2 v_{e} a_{e}\right) \kappa^{2} F_{3}^{Z}
\end{aligned}
$$

with $v_{e}=-\frac{1}{2}+2 \sin ^{2} \theta_{W}, a_{e}=-\frac{1}{2}, \kappa^{-1}=4 \sin ^{2} \theta_{W} \cos ^{2} \theta_{W}\left(Q^{2}+M_{Z}^{2}\right) / Q^{2}$ using $\sin ^{2} \theta_{W}=0.2312$ and $M_{Z}=91.1876 \mathrm{GeV}$. Note that the structure functions in (7) refer to the radiatively corrected ones as presented by the experimentalists. Furthermore, the well-known standard target mass corrections to $F_{2}$ have been taken into account in the medium to large $x$-region for $Q^{2}<100 \mathrm{GeV}^{2}$. Since the experimental extraction of the usual (one-photon exchange) $F_{2}\left(x, Q^{2}\right)$ from $d^{2} \sigma / d x d Q^{2}$ is obviously (parton) model dependent, we have chosen to work with the full NC framework in order to avoid any further dependence on model assumption. However, it turned out that fitting just to $F_{2}\left(x, Q^{2}\right)$ gives very similar results. Furthermore, since the longitudinal structure function $F_{\mathrm{L}}$ contributes sizeably only for large inelasticity $y$, in most of the kinematic range we have $\sigma_{r} \simeq F_{2}$. In addition we have used the fixed target $F_{2}^{p}$ data of SLAC [31, BCDMS [32], E665 [33] and NMC [34] subject to the standard cuts $Q^{2} \geq 4 \mathrm{GeV}^{2}$ and 
$W^{2}=Q^{2}\left(\frac{1}{x}-1\right)+m_{p}^{2} \geq 10 \mathrm{GeV}^{2}$, together with the structure function ratios $F_{2}^{n} / F_{2}^{p}$ of BCDMS [35], E665 [36] and NMC [37]. The data for heavy quark $(c, b)$ production, being theoretically described in the fixed-flavor number factorization scheme by the fully predictive fixed-order ( $\mathrm{LO} / \mathrm{NLO})$ perturbation theory to be discussed below, are taken from [38, 39, 40] for $F_{2}^{c}$ and from [40] for $F_{2}^{b}$. Furthermore the Drell-Yan muon pair production data of E866/NuSea [41] for $d^{2} \sigma^{p N} / d x_{F} d M_{\mu^{+} \mu^{-}}$with $N=p, d$ have been used, as well as their asymmetry measurements [42] for $\sigma^{p d} / \sigma^{p p}$. These data are instrumental in fixing $\bar{d}-\bar{u}$ (or $\bar{d} / \bar{u}$ ). The relevant LO/NLO differential Drell-Yan cross sections can be found in the Appendix of [43], except for eq. (A8) which has to be modified [44, 45] in order to conform with the usual $\overline{\mathrm{MS}}$ convention for the number of gluon polarization states $2(1-\varepsilon)$ in $4-2 \varepsilon$ dimensions. Finally, the Tevatron high- $p_{\mathrm{T}}\left(\right.$ or $E_{\mathrm{T}}$ ) inclusive jet data of D0 [46] and CDF [47] have been used together with the fastNLO [48] package for calculating the relevant cross sections at NLO. It should be mentioned that all these data sets correspond to a total of of 1739 data points.

As already noted, the LO and NLO heavy quark contributions $F_{i}^{c, b}$ are calculated in the FFNS and contribute to the total structure functions as $F_{i}\left(x, Q^{2}\right)=F_{i}^{\text {light }}+F_{i}^{\text {heavy }}$ where 'light' refers to the common $u, d, s$ (anti)quarks and gluon initiated contributions [44], and $F_{i}^{\text {heavy }}=F_{i}^{c}+F_{i}^{b}$. Top quark contributions are negligible. The LO $\mathcal{O}\left(\alpha_{s}\right)$ contributions 1 to $F_{2, \mathrm{~L}}^{h}$, due to the subprocess $\gamma^{*} g \rightarrow h \bar{h}$, have been summarized in [7], and the NLO $\mathcal{O}\left(\alpha_{s}^{2}\right)$ ones are given in [51, 52]. These contributions are gluon $g\left(x, \mu_{F}^{2}\right)$ dominated where the factorization scale should preferably be chosen [53] to be $\mu_{F}^{2}=4 m_{h}^{2}$. As we shall see the resulting predictions are in perfect agreement with all available DIS data on heavy quark production and are futhermore perturbatively stable [53]. Even choosing a very large scale like $\mu_{F}^{2}=4\left(Q^{2}+4 m_{c}^{2}\right)$ leaves the NLO results essentially unchanged [8, 54] in particular at small- $x$. This stability renders attempts to resum supposedly 'large

\footnotetext{
${ }^{1}$ It has become common to consider the $\mathcal{O}\left(\alpha_{s}\right)$ contributions to $F_{\mathrm{L}}^{\text {light }}$ as LO [49]. Therefore the 2-loop $\mathcal{O}\left(\alpha_{s}^{2}\right)$ Wilson coefficients [49] are required for $F_{\mathrm{L}}^{\text {light }}$ at NLO. (Simplified parametrizations of the relevant Wilson coefficients can be found in [50].)
} 
logarithms' $\left(\ln Q^{2} / m_{h}^{2}\right)$ in heavy quark production cross sections superfluous. The heavy quark contributions to the remaining $\mathrm{NC}$ structure functions $F_{2, \mathrm{~L}}^{\gamma \mathrm{Z}}$ and $F_{2, \mathrm{~L}}^{\mathrm{Z}}$ in $(5)$ are quantitatively negligible. Nevertheless, they can be simply obtained from the $\gamma$-exchange contributions $F_{2, L}^{h}$ by substitutions like $e_{q}^{2} \rightarrow 2 e_{q} v_{q}$ and $e_{q}^{2} \rightarrow v_{q}^{2}+a_{q}^{2}$, respectively, where $v_{q}= \pm \frac{1}{2}-2 e_{q} \sin ^{2} \theta_{W}$ and $a_{q}= \pm \frac{1}{2}$ with \pm referring to an up-or down-type quark. The heavy quark contributions to $F_{3}^{\gamma \mathrm{Z}, \mathrm{Z}}$ vanish in LO [55, 56], and are negligibly small in NLO since effectively $F_{3}^{h} \sim h-\bar{h}$ at the relevant large values of $Q^{2}$.

In order to test the dependence of our results on the specific choice of the factorization scheme in NLO, other than the commonly used $\overline{\mathrm{MS}}$ scheme, we also perform our NLO analysis using the deep inelastic scattering (DIS) factorization scheme [25, 26]. Here the $\overline{\mathrm{MS}}$ Wilson coeffients are absorbed into the parton distributions, or more precisely into their evolutions, i.e., into the splitting functions. This transformation to the DIS scheme in NLO is achieved via [44]

$$
\begin{gathered}
P_{\mathrm{NS}}^{(1)} \rightarrow P_{\mathrm{NS}, \mathrm{DIS}}^{(1)}=P_{\mathrm{NS}}^{(1)}+\beta_{0} \Delta C_{2, \mathrm{NS}}^{(1)} \\
\hat{P}^{(1)} \rightarrow \hat{P}_{\mathrm{DIS}}^{(1)}=\hat{P}^{(1)}+\beta_{0} \Delta \hat{C}_{2}^{(1)}-\left[\Delta \hat{C}_{2}^{(1)} \otimes \hat{P}^{(0)}-\hat{P}^{(0)} \otimes \Delta \hat{C}_{2}^{(1)}\right]
\end{gathered}
$$

where

$$
\Delta C_{2, \mathrm{NS}}^{(1)}=-C_{2, \mathrm{NS}}^{(1)} \quad, \quad \Delta \hat{C}_{2}^{(1)}=-\left(\begin{array}{ccc}
C_{2, q}^{(1)} & , & C_{2, g}^{(1)} \\
-C_{2, q}^{(1)} & , & -C_{2, g}^{(1)}
\end{array}\right) .
$$

The light $u, d, s$ quark contributions to $F_{2}^{p}$, for example, in the NLO(DIS) factorization scheme now simply become

$$
F_{2}^{\text {light }}\left(x, Q^{2}\right)=x \sum_{q=u, d, s} e_{q}^{2}\left[q\left(x, Q^{2}\right)+\bar{q}\left(x, Q^{2}\right)\right]_{\mathrm{DIS}} .
$$

The quantitative difference between the $\operatorname{NLO}(\overline{\mathrm{MS}})$ and $\mathrm{NLO}(\mathrm{DIS})$ results will turn out to be rather small. Having obtained the parton distributions $\stackrel{(-)}{q}\left(x, Q^{2}\right)$ DIS and $g\left(x, Q^{2}\right)$ DIS from an explicit NLO analysis of $F_{2}\left(x, Q^{2}\right)$ in the DIS factorization scheme, one can 
transform them to the $\overline{\mathrm{MS}}$ scheme via (see [7], for example)

$$
\begin{aligned}
\stackrel{(-)}{q}\left(x, Q^{2}\right) & =\stackrel{(-)}{q}\left(x, Q^{2}\right)_{\mathrm{DIS}}-a\left[C_{2, q}^{(1)} \otimes(\stackrel{-)}{q})\right. \\
g\left(x, Q^{2}\right) & =g\left(x, Q^{2}\right)_{\mathrm{DIS}}+a\left[C_{2, q}^{(1)} \otimes \Sigma_{\mathrm{DIS}}+C_{2, g}^{(1)} \otimes g_{\mathrm{DIS}}\right]\left(x, Q^{2}\right)+\mathcal{O}\left(a^{2}\right)
\end{aligned}
$$

where

$$
\begin{aligned}
& C_{2, q}^{(1)}(z)=2 \frac{4}{3}\left[\frac{1+z^{2}}{1-z}\left(\ln \frac{1-z}{z}-\frac{3}{4}\right)+\frac{1}{4}(9+5 z)\right]_{+} \\
& C_{2, g}^{(1)}(z)=4 n_{f} \frac{1}{2}\left[\left(z^{2}+(1-z)^{2}\right) \ln \frac{1-z}{z}-1+8 z(1-z)\right]
\end{aligned}
$$

with $n_{f}=3$. This transformation to the $\overline{\mathrm{MS}}$ scheme then allows also for a consistent NLO analysis of heavy quark and Drell-Yan dimuon production processes in the DIS scheme, using their well known theoretical $\overline{\mathrm{MS}}$ expressions, as well as for a consistent comparison of our DIS results with the ones obtained in the $\overline{\mathrm{MS}}$ factorization scheme.

\section{2a. Estimates of uncertainties}

Our evaluation of the parton distribution uncertainties is based on the Hessian method with the Hessian matrix defined via

$$
\Delta \chi^{2}=\chi^{2}-\chi_{0}^{2}=\frac{1}{2} \sum_{i, j,=1}^{d} H_{i j}\left(a_{i}-a_{i}^{0}\right)\left(a_{j}-a_{j}^{0}\right)
$$

where $\chi_{0}^{2}$ is the value of the minimal $\chi^{2}$ characterized by the free fit parameters $a_{i}^{0}$. In our fits we have $d=21$ and $\chi^{2}$ is calculated by adding the total systematic and statistical experimental errors in quadrature. The uncertainties $\Delta a_{i}=a_{i}-a_{i}^{0}$ are constrained by

$$
\Delta \chi^{2} \leq T^{2}
$$

where the tolerance parameter $T$ was chosen to be [57]

$$
T^{2}=T_{1 \sigma}^{2}=\sqrt{2 N} /(1.65)^{2}
$$


i.e. $T \simeq 4.7$ since $N=1739$ is the total number of data points considered in our global fits. The inversion involved in evaluating $\Delta a_{i}$ in (17), subject to the constraint (18), was performed with the help of the normalized eigenvectors [58] of $H_{i j}$ whose iterative calculation followed [59]. The calculation of all the uncertainties presented in our paper was performed according to the master equation (24) of [58] whose particular implication for $\Delta a_{i}$ is specified in eq. (30) of [58]. Our choice for the displacement distance $t$ entering these latter equations was $t=T$, an assumption made in most subsequent publications and analyses. (When comparing our uncertainty results with the ones of CTEQ [2, 58] where $T=10$ has been assumed, we rescale these CTEQ uncertainties according to $\Delta a_{i} \rightarrow 0.47 \Delta a_{i}$ in order to comply with our $T=4.7$ in (19).)

As suggested in [2], we included in our final error analysis only those parameters that are actually sensitive to the input data set chosen, i.e. those parameters which are not close to 'flat' directions in the overall parameter space. With current data, and our functional form (5), 13 such parameters, including $\alpha_{s}$, are identified and are included in our final error analysis; the remaining ill-determined eight polynomical parameters $A_{f}$ and $B_{f}$, with uncertainties of more than $50 \%$, were held fixed.

\section{Quantitative results and very small- $x$ predictions}

A representative comparison of our dynamical LO and $\mathrm{NLO}(\overline{\mathrm{MS}})$ results with the relevant $\operatorname{HERA}(\mathrm{H} 1, \mathrm{ZEUS})$ data on the proton structure function $F_{2}^{p}\left(x, Q^{2}\right)$ is presented in Figs. 1 and 2. Due to our valence-like input, the small- $x$ results $\left(x \lesssim 10^{-2}\right)$ are predictions being entirely generated by the QCD $Q^{2}$-evolutions. This is in contrast to a 'standard fit' where the gluon and sea input distributions in (5) do not vanish as $x \rightarrow 0\left(a_{g, \bar{q}} \lesssim 0\right)$ at $Q_{0}^{2}=2 \mathrm{GeV}^{2}$. For comparison we have also performed such a standard fit shown by

the dashed-dotted curves in Figs. 1 and 2. In both cases the data in Figs. 1 and 2 are well described throughout the whole medium- to small-x region for $Q^{2} \gtrsim 2 \mathrm{GeV}^{2}$ and 
thus perturbative QCD is here fully operative. At $Q^{2}<2 \mathrm{GeV}^{2}$ the theoretical results fall below the data in the very small $-x$ region; this is not unexpected for perturbative leading twist-2 results, since nonperturbative (higher twist) contributions to $F_{2}\left(x, Q^{2}\right)$ will eventually become relevant, even dominant, for decreasing values of $Q^{2}$. It should be emphasized that all of our valence-like input distributions at $\mu_{\mathrm{LO}}^{2}=0.3 \mathrm{GeV}^{2}$ and $\mu_{\mathrm{NLO}}^{2}=0.5 \mathrm{GeV}^{2}$ as well as the ones for the 'standard fit' at $Q_{0}^{2}=2 \mathrm{GeV}^{2}$ are manifestly positive. This is in contrast to negative gluon distributions in the small- $x$ region observed in other standard fits [3, 4, 5]. Furthermore the more restrictive ansatz of the valence-like input distributions at small- $x$ as well as the sizeably larger evolution distance (starting at $Q_{0}<1 \mathrm{GeV}$ ) imply smaller uncertainties concerning the behavior of structure functions in the small- $x$ region than the corresponding results obtained from the common 'standard fits', in particular as $Q^{2}$ increases. This is illustrated in Fig. 3 for the $\mathrm{NLO}(\overline{\mathrm{MS}})$ results where the error bands correspond to a $1 \sigma$ uncertainty. Since our valence-like sea input has a rather small power of $x$, i.e. vanishes only slowly as $x \rightarrow 0$, the uncertainties of the sea dominated $F_{2}\left(x, Q^{2}\right)$ turn out to be not too different from the standard fit where the sea increases as $x \rightarrow 0$ (negative power of $x$ ) already at the input scale $Q_{0}^{2}=2 \mathrm{GeV}^{2}$. Notice that the uncertainties generally decrease as $Q^{2}$ increases due to the QCD $Q^{2}$-evolutions [57, 58.

Our $\operatorname{NLO}(\overline{\mathrm{MS}})$ valence-like input distributions at $Q_{0}^{2}=\mu_{\mathrm{NLO}}^{2}=0.5 \mathrm{GeV}^{2}$ together with their $1 \sigma$ uncertainties are shown in Fig. 4. They have been parametrized according to (5) with the parameters given in Table 1.2 For comparison the GRV98 input [8] is displayed as well, which turns out to be very similar except for the gluon which peaks at a slightly larger value of $x$. However, such differences are merely within a $2 \sigma$ band of our new results. The valence-like gluon input at low $Q^{2}<1 \mathrm{GeV}^{2}$ in Fig. 4 implies

\footnotetext{
${ }^{2}$ It should be mentioned that there is a correlation between the (chosen) value of $\alpha_{s}\left(M_{\mathrm{Z}}^{2}\right)$ and the resulting values of the valence-like input scales $\mu_{\mathrm{LO}, \mathrm{NLO}}$ which increase with $\alpha_{s}\left(M_{\mathrm{Z}}^{2}\right)$. Since we did not want to fix $\alpha_{s}\left(M_{\mathrm{Z}}^{2}\right)$ at the LEP value of 0.118 , we performed fits for various fixed values of $\mu$ by imposing a valence-like input structure $\left(a_{g, \bar{u}+\bar{d}}>0\right)$ and keeping $\alpha_{s}$ as a free fit parameter. Then we fixed the best choice for $\mu\left(\mu_{\mathrm{LO}}^{2}=0.3 \mathrm{GeV}^{2}, \mu_{\mathrm{NLO}}^{2}=0.5 \mathrm{GeV}^{2}\right)$ and performed the final precision fits and error analyses.
} 
a far stronger constrained gluon distribution at larger values of $Q^{2}$ as compared to a gluon density obtained from a 'standard fit' with a conventional non-valence-like input at $Q^{2}>1 \mathrm{GeV}^{2}$ as can be seen in Fig. 5. As already mentioned, this is in contrast to the sea distribution $\bar{u}+\bar{d}$ in Fig. 5 where the valence-like sea input in Fig. 4 vanishes very slowly as $x \rightarrow 0$ and thus is similarly increasing with decreasing $x$ down to $x \simeq 0.01$ as the sea input obtained by a standard fit. Therefore the $1 \sigma$ uncertainty band of our dynamically predicted sea distributions at larger values of $Q^{2}$ in Fig. 5 is only marginally smaller than the corresponding one of the standard fit. The relevant input parameters of our 'standard fit' can be found in Table 2. As expected for the dynamical fit, starting from a low input scale with valence-like distributions, $\alpha_{s}\left(M_{\mathrm{Z}}^{2}\right)$ in Table 1 is somewhat stronger constrained due to the larger evolution distance than the corresponding result of the standard $\mathrm{NLO}(\overline{\mathrm{MS}})$ fit in Table 2. Keeping in mind that our stated errors always refer to $1 \sigma$ uncertainties, our standard fit error of 0.0021 for $\alpha_{s}\left(M_{\mathrm{Z}}^{2}\right)$ in Table 2 is compatible with the $2 \sigma$ uncertainty also stated in the literature (see, e.g., [2] and the discussion in [3]). It should be furthermore mentioned that our $\operatorname{NLO}(\overline{\mathrm{MS}})$ results for $\alpha_{s}\left(M_{Z}^{2}\right)$ in Table 1 and 2 are, within about a $1 \sigma$ uncertainty, also compatible with the ones obtained from analyzing only DIS structure functions (for a recent summary, see [60]).

At this point it should be mentioned that the standard CTEQ [2] fit resulted, very surprisingly, in a valence-like input gluon distribution at a scale as large as $Q_{0}^{2}=m_{c}^{2} \simeq 1.7$ $\mathrm{GeV}^{2}$. Thus this CTEQ6 gluon distribution is expected to be similarly tightly constrained at $Q^{2}>Q_{0}^{2}$ as our dynamical results starting from a valence-like input at $Q_{0}^{2}=0.5 \mathrm{GeV}^{2}$. That this is indeed the case is illustrated in Fig. 6 where the $1 \sigma$ uncertainties of the CTEQ6 gluon [2] are similar in size to our dynamical results, whereas a common 'standard fit' (being based on an increasing input distribution as $x \rightarrow 0$ ) results in a sizeably larger uncertainty. The situation is different for the sea distribution in the small- $x$ region; here the CTEQ6 input at $Q_{0}^{2}=m_{c}^{2}$ increases at $x \rightarrow 0$ as expected, and thus the $1 \sigma$ uncertainty is comparable to our 'standard fit' result as shown in Fig. 7 - both being larger than the 
$1 \sigma$ uncertainty obtained from our dynamical fit based on valence-like inputs at $Q_{0}^{2}=0.5$ $\mathrm{GeV}^{2}$.

The heavy charm and bottom quark contributions to $F_{2}$ at LO and $\mathrm{NLO}(\overline{\mathrm{MS}})$ are compared with recent HERA data in Figs. 8 and 9. The impressive agreement with present measurements for $F_{2}^{c}$ and $F_{2}^{b}$ illustrates that the $n_{f}=3$ FFNS is entirely reliable. As already discussed in Sec. 2, the NLO results are rather insensitive to the chosen factorization scale $\mu_{F}\left(\mu_{F}^{2}=4 m_{h}^{2}\right.$ or $\left.\mu_{F}^{2}=Q^{2}+4 m_{h}^{2}\right)$. Again the $1 \sigma$ uncertainties of these dynamical predictions are distinctly smaller than the ones implied by the standard fit. It should be furthermore reemphasized that within the FFNS heavy quarks $(h=$ $c, b, t)$ are always produced as final states in fixed order perturbation theory via hard production processes initiated by the light partons of the nucleon $(u, d, s$ quarks and the gluon $g$ ). The perturbative stability of heavy quark production [53] as well as the agreement with experiment in Figs. 8 and 9 even at $Q^{2} \gg m_{h}^{2}$ indicate that there is no need to resum supposedly 'large logarithms' $\left(\ln Q^{2} / m_{h}^{2}\right)$, which is of course in contrast to genuine collinear logarithms appearing in light (massless) quark and gluon hard scattering processes. Therefore only the $n_{f}=3$ light $u, d, s$ quark flavors and gluons constitute the 'intrinsic' genuine partons of the proton and the heavy $c, b, t$ quark flavors should not be included in the parton structure of the nucleon, not even at $Q^{2} \gg m_{h}^{2}$ [53]. However, somewhat dissenting views were recently summarized in [61].

The measurements of Drell-Yan dilepton production in $p p$ and $p d$ collisions [41, 42] are instrumental in fixing $\Delta=\bar{d}-\bar{u}$ (or $\bar{d} / \bar{u}$ ) [62]. In Fig. 10 we display our dynamical $\operatorname{NLO}(\overline{\mathrm{MS}})$ result for $\sigma^{p d} / 2 \sigma^{p p}$ together with the $\pm 1 \sigma$ uncertainty band as well as the previous GRV98 result which agree in the statistically relevant $x$-region, with $x_{2}$ referring to the average fractional momentum of the target partons. Note that $\sigma^{p N} \equiv d^{2} \sigma^{p N} / d x_{F} d M_{\mu^{+} \mu^{-}}^{2}$ with $x_{F}=x_{1}-x_{2}$. In LO $\sigma^{p N} \sim \sum_{u, d, s} e_{q}^{2}\left[q\left(x_{1}\right) \bar{q}\left(x_{2}\right)+q\left(x_{2}\right) \bar{q}\left(x_{1}\right)\right]$ where $x_{1}$ and $x_{2}$ refer to the Bjorken $-x$ of the quarks in the beam $(p)$ and nucleon target $(N)$, respectively. Experimentally $x_{F}>0\left(x_{1}>x_{2}\right)$ and consequently the Drell-Yan cross section is dominated 
by the annihilation of a beam quark with a target antiquark. For $x_{1} \gg x_{2}$ one obtains $\sigma^{p d} / 2 \sigma^{p p} \simeq \frac{1}{2}\left[1+\bar{d}\left(x_{2}\right) / \bar{u}\left(x_{2}\right)\right]$ at a scale $Q^{2} \equiv M_{\mu^{+} \mu^{-}}^{2}$ in $\bar{q}\left(x_{2}, Q^{2}\right)$.

Finally the $p \bar{p}$ Tevatron high $-p_{\mathrm{T}}$ (or $E_{\mathrm{T}}$ ) inclusive jet data [46, 47] are compared in Fig. 11 with our dynamical LO and $\mathrm{NLO}(\overline{\mathrm{MS}})$ results, as well as with the ones of CTEQ6 [2]. The small $1 \sigma$ error bands are almost invisible on the huge logarithmic scale used. Our NLO result almost coincides with the one of CTEQ. There is a clear improvement at NLO as compared to LO which falls slightly below the data at $p_{\mathrm{T}} \lesssim 300 \mathrm{GeV}$. Nevertheless the LO high $-p_{\mathrm{T}}$ fit corresponds to $\chi^{2} /$ dof $\simeq 1$ which is only twice as large as at NLO.

As discussed in Sec. 2 we have explicitly used for our analysis the experimentally directly measured 'reduced' DIS cross sections (7) which, for not too large values of $Q^{2}$, are dominated by the one-photon exchange cross section $\sigma_{r}=F_{2}-\left(y^{2} / Y_{+}\right) F_{\mathrm{L}}$ where $y=Q^{2} / x s$. The importance of using this quantity has recently been emphasized [63] since the effect of $F_{\mathrm{L}}$ becomes increasingly relevant as $x$ decreases at a given $Q^{2}$ where $y$ increases. This is seen in the data as a flattening of the growth of $\sigma_{r}\left(x, Q^{2}\right)$ as $x$ decreases to very small values, at fixed $Q^{2}$, leading eventually to a turnover (cf. Fig. 12). At the lower values of $Q^{2}$ in Fig. 12 it was not possible in 63] to reproduce this turnover at NLO. This was mainly due to the negative longitudinal cross section (negative $F_{\mathrm{L}}\left(x, Q^{2}\right)$ ) encountered in [63]. Since all of our cross sections and structure functions are manifestly positive throughout the whole kinematic region considered, our dynamical NLO $(\overline{\mathrm{MS}})$ results in Fig. 12 are in good agreement with all small- $x$ HERA measurements [28, 29]. For completeness we compare in Fig. 13 our dynamical (leading twist) $\operatorname{NLO}(\overline{\mathrm{MS}})$ predictions for $F_{\mathrm{L}}\left(x, Q^{2}\right)$ with a representative selection of (partly prelinimary) H1 data [28, 64] at fixed $W \simeq 276 \mathrm{GeV}$. The standard fit result with its sizeably larger $\pm 1 \sigma$ error band is, for comparison, shown as well. Our NLO results for $F_{\mathrm{L}}$, being gluon dominated in the small- $x$ region, are in full agreement with present measurements, which is in contrast to expectations [3, 63] based on negative parton distributions and structure functions at small $x$. To illustrate the manifest positive definiteness of our dynamically generated 
structure functions we show $F_{\mathrm{L}}\left(x, Q^{2}\right)$ in Fig. 13 down to $Q^{2}=1 \mathrm{GeV}^{2}$ although a leading twist-2 prediction should not be confronted with data below, say, $2 \mathrm{GeV}^{2}$.

As our parameter-free small- $x$ predictions for parton distributions at $x<10^{-2}$ are entirely of QCD-dynamical origin and depend rather little on the detailed input parameters at $x \gtrsim 10^{-2}$, it is interesting to study these predictions in kinematic regions not accessible by present DIS experiments. Of particular interest are, as already emphasized in the Introduction, calculations of weak $\stackrel{(-)}{\nu} N$ cross sections of ultrahigh energy cosmic neutrinos $[12,15,65-69]$ which afford a (reliable) knowledge of parton distributions at the weak scale $Q^{2}=M_{W}^{2}$ down to $x \simeq 10^{-9}$ for highest energies $E_{\nu} \simeq 10^{12} \mathrm{GeV}$. This requires extrapolations into the unmeasured small- $x$ region $x<10^{-3}$. Since $F_{2}^{p}\left(x, Q^{2}\right)$ is, in the very small- $x$ region, dominated by $\bar{u}\left(x, Q^{2}\right)$ and $\bar{d}\left(x, Q^{2}\right)$, as are the CC neutrino(isoscalar) nucleon cross sections, the $F_{2}^{p}$ structure function can be utilized for estimating the magnitude of uncertainties of the predictions in the extreme small- $x$ region which are shown in Fig. 14. At $Q^{2}=M_{W}^{2}$ our dynamical NLO predictions correspond to a $\pm 1 \sigma$ uncertainty of about $\pm 7 \%$ at $x=10^{-9}$ whereas the uncertainty of the extrapolation of a standard fit is about twice as large. At smaller scales the uncertainties obviously increase as illustrated in Fig. 14 at $Q^{2}=100 \mathrm{GeV}^{2}$. Taking into account previous extrapolation ambiguities [8], one can conclude [12] that the dynamically predicted small- $x$ parton distributions allow neutrino-nucleon cross sections to be calculable with an accuracy of about $10 \%$ at highest cosmic neutrino energies. It should be mentioned that an ad hoc fixed power law of $x$ extrapolation of the standard CTEQ6.5 structure functions [70] to $x=10^{-8}$ at $Q^{2}=M_{W}^{2}$ [71] lies, accidentally, only about $10 \%$ below our dynamical NLO prediction in Fig. 14. On the other hand, an alternative parametrization [71] of present HERA(ZEUS) data which is not QCD oriented but based on analyticity and unitarity gives, when extrapolated to $x=10^{-8}$, a factor of about 6 smaller a value for $F_{2}^{p}\left(10^{-8}, M_{W}^{2}\right)$ than our prediction in Fig. 14. Since the perturbative dynamical QCD predictions for the small $-x$ behavior of structure functions down to $x=10^{-5}$ proved to 
be in agreement with later HERA measurements as discussed in the Introduction, it is hard to imagine that perturbative QCD dynamics and evolutions should become entirely inappropriate at $x=10^{-8}$ to $10^{-9}$ at even much larger scales.

In order to test the dependence of our results on the specific choice of the factorization scheme in NLO, we have repeated our dynamical analysis in the DIS factorization scheme as outlined in Sec. 2. Since the (light) parton distributions in the DIS scheme are defined via the $F_{2}$ structure function in (12), it is not very surprising that, in contrast to the DIS parton distributions themselves [72], the results for physical observables directly related to DIS structure functions are very similar. Indeed the results in the DIS factorization scheme for $F_{2}^{p}\left(x, Q^{2}\right)$ in Figs. 1 to 3 and 14 are practically indistinguishable from the ones in the $\overline{\mathrm{MS}}$ scheme, as are the results for $F_{2}^{c, b}\left(x, Q^{2}\right)$ in Figs. 8 and 9 and the ones for $\sigma_{r}\left(x, Q^{2}\right)$ and $F_{\mathrm{L}}\left(x, Q^{2}\right)$ in Figs. 12 and 13, respectively. Differences become visible only for processes which are not directly related to DIS structure functions such as DrellYan cross sections but there the differences lie well within the $\pm 1 \sigma$ uncertainty of the $\operatorname{NLO}(\overline{\mathrm{MS}})$ results as illustrated in Fig. 10 for the DY-asymmetry. The parameters for the input parton distributions in the DIS scheme and the corresponding $\alpha_{s}\left(M_{Z}^{2}\right)$ can be found in Table 1. It should be furthermore emphasized that our results and predictions are also stable to within less than about $20 \%$ when compared to previous analyses and fits. This is illustrated in Fig. 15 for our present dynamical $\mathrm{NLO}(\overline{\mathrm{MS}})$ results when compared with our previous GRV98 results [8]. The situation is similar for more recent and previous standard CTEQ and MRST parton distributions for their relevant ranges in $x$, and holds also by comparing CTEQ and MRST distributions with each other [2, 3, 57, 70, 73, 74]. It should be emphasized that heavy quark mass effects have always been fully taken into account in our previous [7, 8] and present analyses. This is in contrast to the previous CTEQ6 analysis [2] where charm has been treated in the zero-mass approximation. The recent inclusion of finite charm mass effects in CTEQ6.5 [70] reduces the charm contribution to $F_{2}\left(x, Q^{2}\right)$ which is compensated by larger $u=u_{v}+\bar{u}$ and $d=d_{v}+\bar{d}$ distributions at 
small $x$ as compared to CTEQ6 [2]. That such an 'enhancement' has always been present in our dynamical $u$ and $d$ distributions is illustrated in Fig. 16, since our present and previous (cf. Fig. 15) distributions differ very little from the CTEQ6.5 ones. Therefore our predicted hadronic $W^{ \pm} / Z^{0}$ production cross sections, for example, at Tevatron and LHC are similar to the 'enhanced' ones observed in [70].

Of course more recent parton distributions have a higher precision due to the higher statistics of the data, but we have not experienced essential qualitative and quantitative changes during the past decade. It is reassuring to see that our knowledge of the fundamental partonic structure of matter has essentially remained unchanged over the past years.

\section{Summary and Conclusions}

Utilizing recent DIS measurements and data on Drell-Yan dilepton and high- $E_{\mathrm{T}}$ inclusive jet production, we have redone a previous [8] global fit for the dynamical parton distributions of the nucleon in the LO and NLO of perturbative QCD. The small- $x\left(x \lesssim 10^{-2}\right)$ structure of dynamical parton distributions is generated entirely radiatively from valencelike, manifestly positive, input distributions at an optimally chosen input scale $Q_{0}<1$ $\mathrm{GeV}$. The NLO results are stable with respect to a different choice of the factorization scheme ( $\overline{\mathrm{MS}}$ versus DIS). The predictions for the longitudinal structure function $F_{\mathrm{L}}\left(x, Q^{2}\right)$ at small $x$, for example, are positive throughout the whole kinematic region considered, in agreement with (partly preliminary) data. We have augmented our analyses with an appropriate uncertainty analysis and found that the newly determined dynamical distributions are compatible with the former [7, 8] ones, where heavy quark mass effects have always been fully taken into account. The stability of these results guarantees a reliable calculation of cross sections for, e.g., heavy quark, $W^{ \pm}, Z^{0}$, and high- $p_{\mathrm{T}}$ jet production at hadron colliders like Tevatron and in particular LHC. 
Our dynamical distributions have also been compared with conventional ('standard') ones obtained from non-valence-like positive input distributions at some arbitrarily chosen higher input scale $Q_{0}>1 \mathrm{GeV}$. For this purpose we have performed a 'standard fit' as well, assuming $Q_{0}^{2}=2 \mathrm{GeV}^{2}$. The uncertainties of these latter distributions are, as expected, larger, in particular in the present (experimentally) unexplored extremely small- $x$ region relevant for evaluating ultrahigh energy neutrino-nucleon cross sections in astrophysical applications. Here we provide predictions down to $x \simeq 10^{-9}$ at the weak scale $Q^{2}=M_{W}^{2}$ as required [12, 65, 66] for highest cosmic neutrino energies of $10^{12} \mathrm{GeV}$. These predictions are strongly constrained within the dynamical parton model and are entirely of QCD-dynamical origin in the very small- $x$ region. Furthermore, as mentioned in the Introduction, previous predictions [6, 9] for the small- $x$ region based on the dynamical parton model and the data available at that time were subsequently confirmed [10, 11] at HERA. The presently available very precise small- $x$ data [28, 29] utilized here allows us to be quite confident about the reliability of our improved small- $x$ predictions within the framework of the successful dynamical parton model.

A FORTRAN package (grid) containing our new dynamical LO, NLO $(\overline{\mathrm{MS}})$, and $\mathrm{NLO}(\mathrm{DIS})$ parton densities, the light $(u, d, s ; g) F_{2}^{\text {light }}\left(x, Q^{2}\right)$ as well as $F_{2}^{c, b}\left(x, Q^{2}\right)$, calculated in the fixed order FFNS, can be obtained by electronic mail or on request. The $\mathrm{NLO}(\overline{\mathrm{MS}})$ uncertainty estimates will be also included.

\section{Acknowledgements}

We thank J. Blümlein for helpful discussions. This work has been supported in part by the 'Bundesministerium für Bildung und Forschung', Berlin/Bonn. 


\section{References}

[1] H.L. Lai et al., CTEQ Collab., Eur. Phys. J. C12, 375 (2000)

[2] J. Pumplin et al., CTEQ Collab., JHEP 07 (2002) 012

[3] A.D. Martin, R.G. Roberts, W.J. Stirling, R.S. Thorne, Eur. Phys. J. C23, 73 (2002)

[4] J. Breitweg et al., ZEUS Collab., Eur. Phys. J. C7, 609 (1999)

[5] S. Chekanov et al., ZEUS Collab., Phys. Rev. D67, 012007 (2003)

[6] M. Glück, E. Reya, A. Vogt, Z. Phys. C53, 127 (1992); Phys. Lett. B306, 391 (1993)

[7] M. Glück, E. Reya, A. Vogt, Z. Phys. C67, 433 (1995)

[8] M. Glück, E. Reya, A. Vogt, Eur. Phys. J. C5, 461 (1998)

[9] M. Glück, E. Reya, A. Vogt, Z. Phys. C48, 471 (1990)

[10] I. Abt et al., H1 Collab., Nucl. Phys. B407, 515 (1993)

[11] M. Derrick et al., ZEUS Collab., Phys. Lett. B316, 412 (1993)

[12] M. Glück, S. Kretzer, E. Reya, Astropart. Phys. 11, 327 (1999)

[13] J.G. Learned, K. Mannheim, Annu. Rev. Nucl. Part. Sci. 50, 679 (2000)

[14] P. Bhattacharjee, G. Sigl, Phys. Rept. 327, 109 (2000)

[15] F. Halzen, Phys. Rept. 333, 349 (2000); Eur. Phys. J. C46, 669 (2006)

[16] D. Diakonov et al., Nucl. Phys. B480, 341 (1996); Phys. Rev. D56, 4069 (1997)

[17] K. Goeke et al., Acta Phys. Polon. B32, 1201 (2001)

[18] H. Weigel, L. Gamberg, H. Reinhardt, Phys. Lett. B399, 287 (1997) 
[19] M. Wakamatsu and T. Kubota, Phys. Rev. D60, 034020 (1999)

[20] M. Wakamatsu, Phys. Rev. D67, 034005 (2003); Phys. Lett. B646, 24 (2007)

[21] C. Bourrely, J. Soffer, F. Bucella, Eur. Phys. J. C23, 487 (2002); C41, 327 (2005)

[22] R.S. Bhalerao, Phys. Lett. B380, 1 (1996); Phys. Rev. C63, 025208 (2001)

[23] R.S. Bhalerao, N.G. Kelkar, B. Ram, Phys. Lett. B476, 285 (2000)

[24] Y.-J. Zhang, B.-S. Zou, Phys. Lett. B528, 228 (2002)

[25] G. Altarelli, R.K. Ellis, G. Martinelli, Nucl. Phys. B143, 521 (1978); B146, 544 (1978) [Erratum]

[26] G. Altarelli, R.K. Ellis, G. Martinelli, Nucl. Phys. B157, 461 (1979)

[27] M. Glück, E. Reya, Mod. Phys. Lett. A22, 351 (2007)

[28] C. Adloff et al., H1 Collab., Eur. Phys. J. C13, 609 (2000); C19, 269 (2001); C21, 33 (2001); C30, 1 (2003)

[29] S. Chekanov et al., ZEUS Collab., Eur. Phys. J. C21, 443 (2001)

[30] M. Klein, T. Riemann, Z. Phys. C24, 151 (1984)

[31] L.W. Whitlow et al., Phys. Lett. B282, 475 (1992)

[32] A.C. Benvenuti et al., BCDMS Collab., Phys. Lett. B223, 485 (1989)

[33] M.R. Adams et al., E665 Collab., Phys. Rev. D54, 3006 (1996)

[34] M. Arneodo et al., NMC Collab., Nucl. Phys. B483, 3 (1997)

[35] A.C. Benvenuti et al., BCDMS Collab., Phys. Lett. B237, 599 (1990)

[36] M.R. Adams et al., E665 Collab., Phys. Rev. Lett. 75, 1466 (1995) 
[37] M. Arneodo et al., NMC Collab., Nucl. Phys. B487, 3 (1997)

[38] S. Chekanov et al., ZEUS Collab., Phys. Rev. D69, 012004 (2004)

[39] C. Adloff et al., H1 Collab., Phys. Lett. B528, 199 (2002)

[40] A. Atkas et al., H1 Collab., Eur. Phys. J. C40, 349 (2005); C45, 23 (2006)

[41] J. Webb, P.E. Reimer, E866/NuSea Collab., hep-ex/0302019 (private communication)

[42] R.S. Towell et al., E866/NuSea Collab., Phys. Rev. D64, 052002 (2001)

[43] P.J. Sutton et al., Phys. Rev. D45, 2349 (1992)

[44] W. Furmanski, R. Petronzio, Z. Phys. C11, 293 (1982)

[45] M. Glück, E. Reya, A. Vogt, Phys. Lett. B285, 285 (1992)

[46] B. Abbott et al., D0 Collab., Phys. Rev. Lett. 86, 1707 (2001)

[47] A. Abulencia et al., CDF Collab., Phys. Rev. D74, 071103 (2006)

[48] T. Kluge, K. Rabbertz, M. Wobisch, fastNLO, hep-ph/0609289

[49] E.B. Zijlstra, W.L. van Neerven, Nucl. Phys. B383, 525 (1992)

[50] S. Moch, J.A.M. Vermaseren, A. Vogt, Phys. Lett. B606, 123 (2005)

[51] E. Laenen et al., Nucl. Phys. B392, 162 (1993)

[52] S. Riemersma, J. Smith, W.L. van Neerven, Phys. Lett. B347, 143 (1995)

[53] M. Glück, E. Reya, M. Stratmann, Nucl. Phys. B422, 37 (1994)

[54] A. Vogt, DESY 96-012 (hep-ph/9601352), Proceedings of DIS '96, Rome, April 1996, eds. G. D’Agostini, A. Nigro (World Scientific 1997), p. 254 
[55] J.P. Leveille, T. Weiler, Nucl. Phys. B147, 147 (1979)

[56] M. Glück, R.M. Godbole, E. Reya, Z. Phys. C38, 441 (1988); C39, 590 (1988) [Erratum]

[57] A.D. Martin et al., Eur. Phys. J. C28, 455 (2003)

[58] J. Pumplin et al., Phys. Rev. D65, 014013 (2002)

[59] J. Pumplin, D.R. Stump, W.K. Tung, Phys. Rev. D65, 014011 (2002)

[60] J. Blümlein, DIS 2007 (Munich, April 2007), arXiv:0706.2430

[61] A. Vogt, DIS 2007 (Munich, April 2007), arXiv:0707.4106, and references therein

[62] P.E. Reimer, arXiv:0704.3621

[63] A.D. Martin, W.J. Stirling, R.S. Thorne, Phys. Lett. B635, 305 (2006)

[64] E.M. Lobodzinska, H1 Collab., DIS 2004 (Strbske Pleso, Slovakia), hep-ph/0311180; T. Lastovicka, Eur. Phys. J. C33, s388 (2004)

[65] G.M. Frichter, D.W. McKay, J.P. Ralston, Phys. Rev. Lett. 74, 1508 (1995); 77, 4107 (1996) [Erratum]

[66] R. Gandhi, C. Quigg, M.H. Reno, I. Sarcevic, Astropart. Phys. 5, 81 (1996); Phys. Rev. D58, 093009 (1998)

[67] K.S. Capelle, J.W. Cronin, G. Parente, E. Zas, Astropart. Phys. 8, 321 (1998)

[68] J.L. Feng, P. Fisher, F. Wilczek, T.M. Yu, Phys. Rev. Lett. 88, 161102 (2002)

[69] K. Giesel, J.-H. Jureit, E. Reya, Astropart. Phys. 20, 335 (2003)

[70] W.K. Tung et al., CTEQ Collab., JHEP 02(2007)053

[71] E.L. Berger, M.M. Block, C.-I. Tan, Phys. Rev. Lett. 98, 242001 (2007) 
[72] M. Glück, C. Pisano, E. Reya, Eur. Phys. J. C50, 29 (2007)

[73] J. Pumplin, DIS 05 (Madison, April 2005), hep-ph 0507093

[74] J. Huston et al., JHEP 06(2005)080 


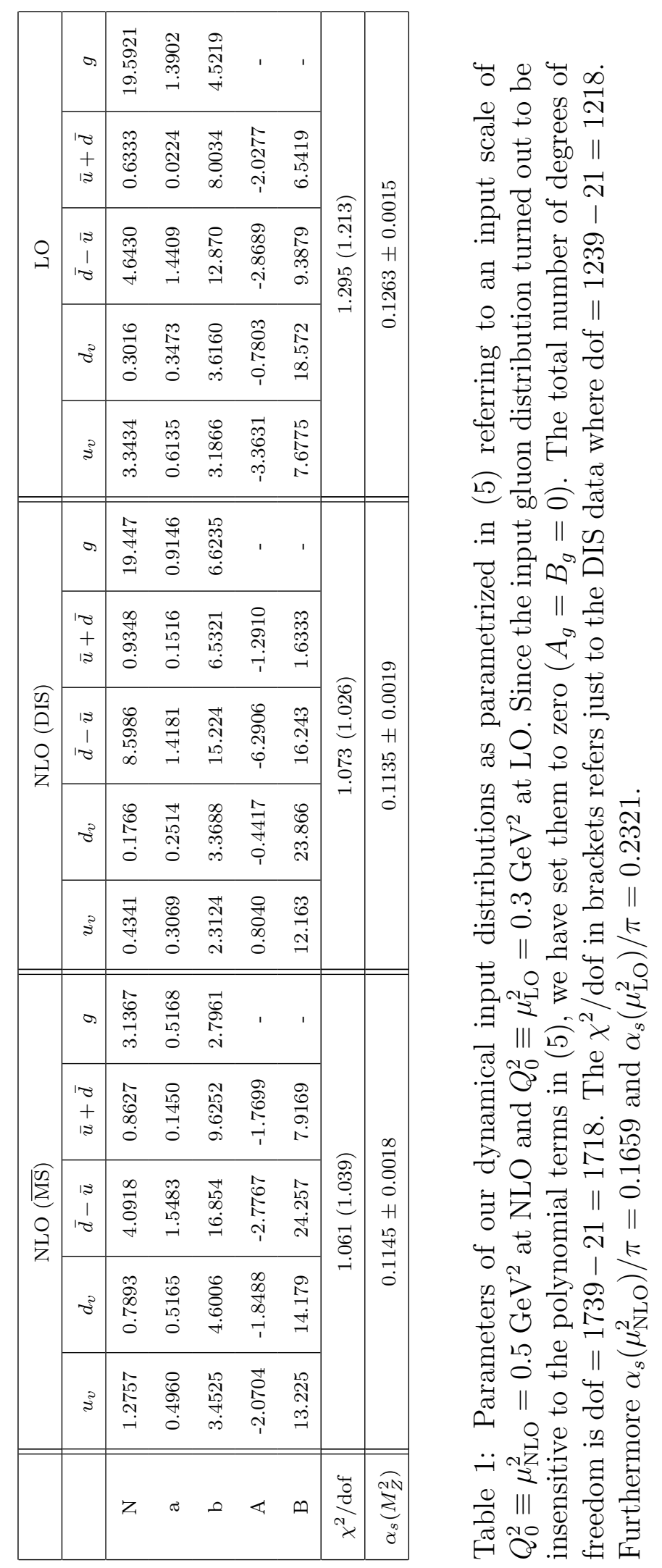




\begin{tabular}{|c||c|c|c|c|c|}
\hline \multicolumn{1}{|c||}{} & \multicolumn{5}{c|}{$\mathrm{NLO}(\overline{\mathrm{MS}})$} \\
\hline & $u_{v}$ & $d_{v}$ & $\bar{d}-\bar{u}$ & $\bar{u}+\bar{d}$ & $g$ \\
\hline $\mathrm{N}$ & 0.5889 & 0.2585 & 7.2847 & 0.2295 & 1.3667 \\
$\mathrm{a}$ & 0.3444 & 0.2951 & 1.2773 & -0.1573 & -0.1050 \\
$\mathrm{~b}$ & 3.7312 & 4.8682 & 18.756 & 8.8819 & 3.3358 \\
$\mathrm{~A}$ & -0.1740 & -1.0552 & -6.3187 & 0.8704 & - \\
$\mathrm{B}$ & 17.997 & 26.536 & 18.306 & 8.2179 & - \\
\hline$\chi^{2} / \mathrm{dof}$ & \multicolumn{5}{c|}{$1.016(0.955)$} \\
\hline$\alpha_{s}\left(M_{Z}^{2}\right)$ & \multicolumn{5}{|c|}{$0.1178 \pm 0.0021$} \\
\hline
\end{tabular}

Table 2: As Table 1 but for the input parameters in (5) of the NLO standard fit at an input scale $Q_{0}^{2}=2 \mathrm{GeV}^{2}$. 

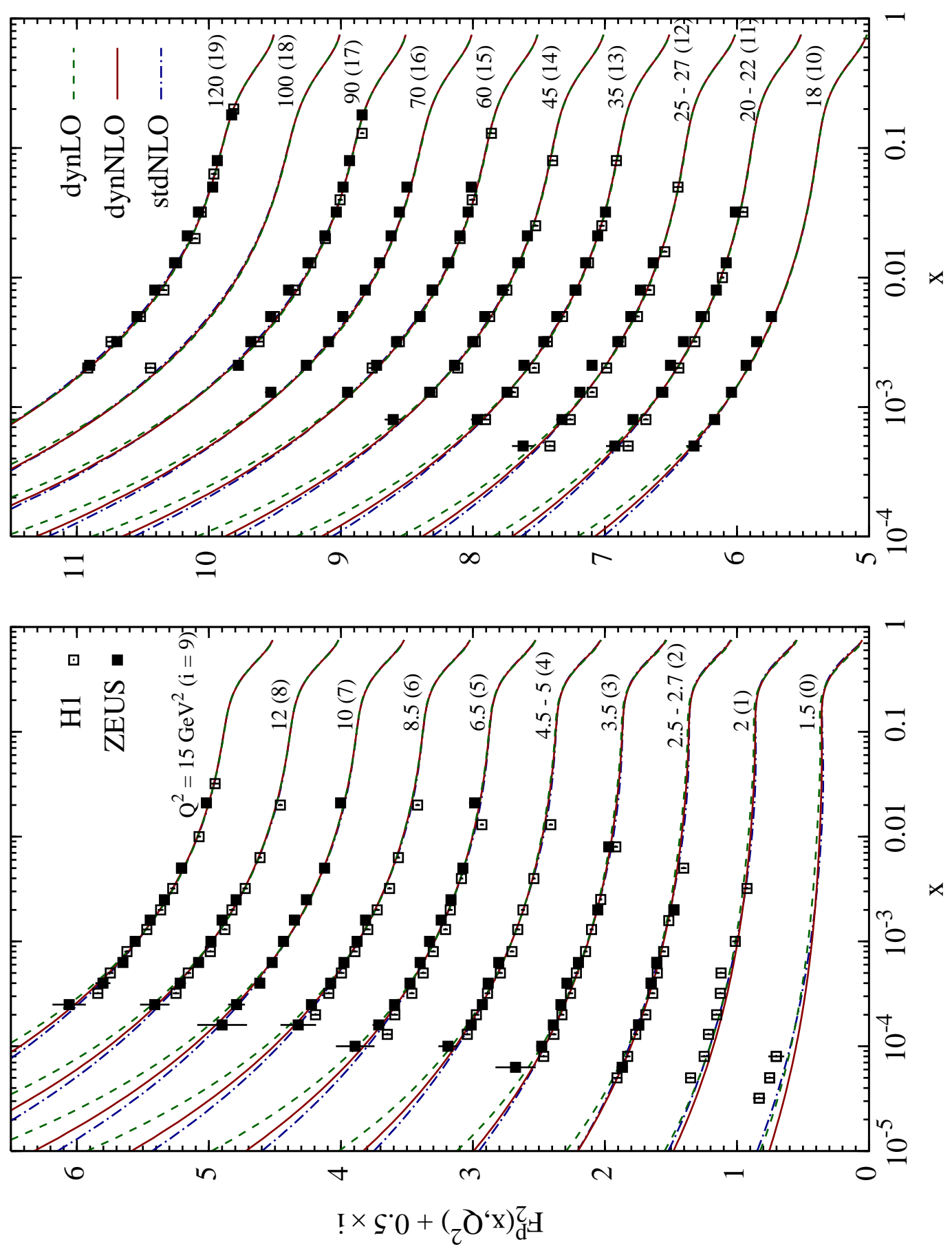

Figure 1: Comparison of our dynamical (dyn) LO and $\mathrm{NLO}(\overline{\mathrm{MS}})$ as well as standard (std) NLO small- $x$ results for $F_{2}^{p}\left(x, Q^{2}\right)$ with HERA data for $Q^{2} \geq 1.5 \mathrm{GeV}^{2}$ [28, 29]. The parameters of the valence-like input distributions for the dynamical predictions are given in Table 1 and the ones for the standard results in Table 2. To ease the graphical presentation we have plotted $F_{2}^{p}\left(x, Q^{2}\right)+0.5 \times i\left(Q^{2}\right)$ with $i\left(Q^{2}\right)$ indicated in parentheses in the figure for each fixed value of $Q^{2}$. 

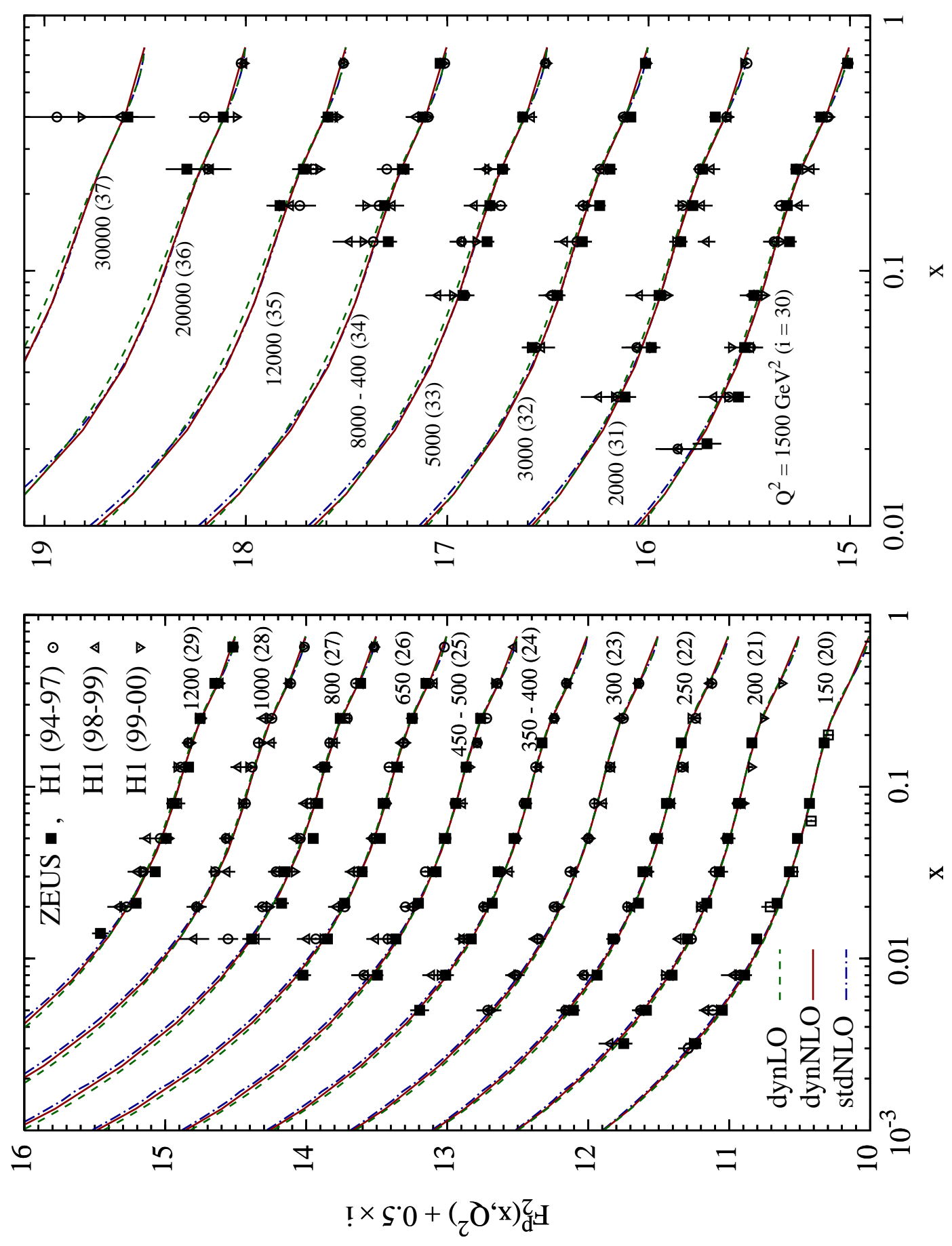

Figure 2: As in Fig. 1 but for large values of $Q^{2}$ and larger $x$. 


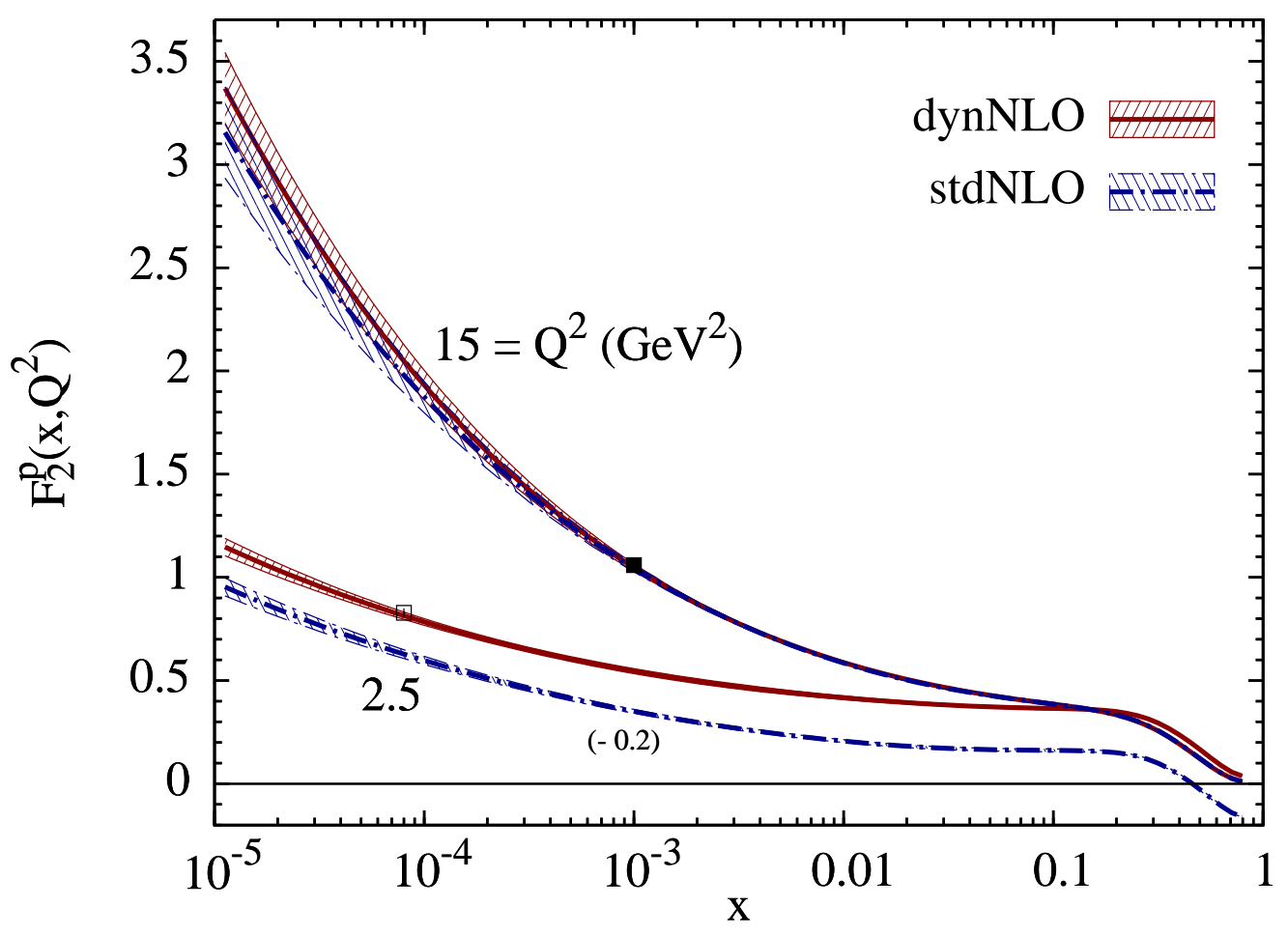

Figure 3: Typical $\pm 1 \sigma$ uncertainty bands of our dynamical and standard $\mathrm{NLO}(\overline{\mathrm{MS}})$ results in Fig. 1 for two representative values of $Q^{2}$. To ease the visibility of the two error bands at $Q^{2}=2.5 \mathrm{GeV}^{2}$ we have subtracted 0.2 from the stdNLO result as indicated. For illustration two $\mathrm{H} 1$ and ZEUS data points from Fig. 1 with their almost invisible errors are shown as well at $Q^{2}=2.5$ and $15 \mathrm{GeV}^{2}$, respectively. 


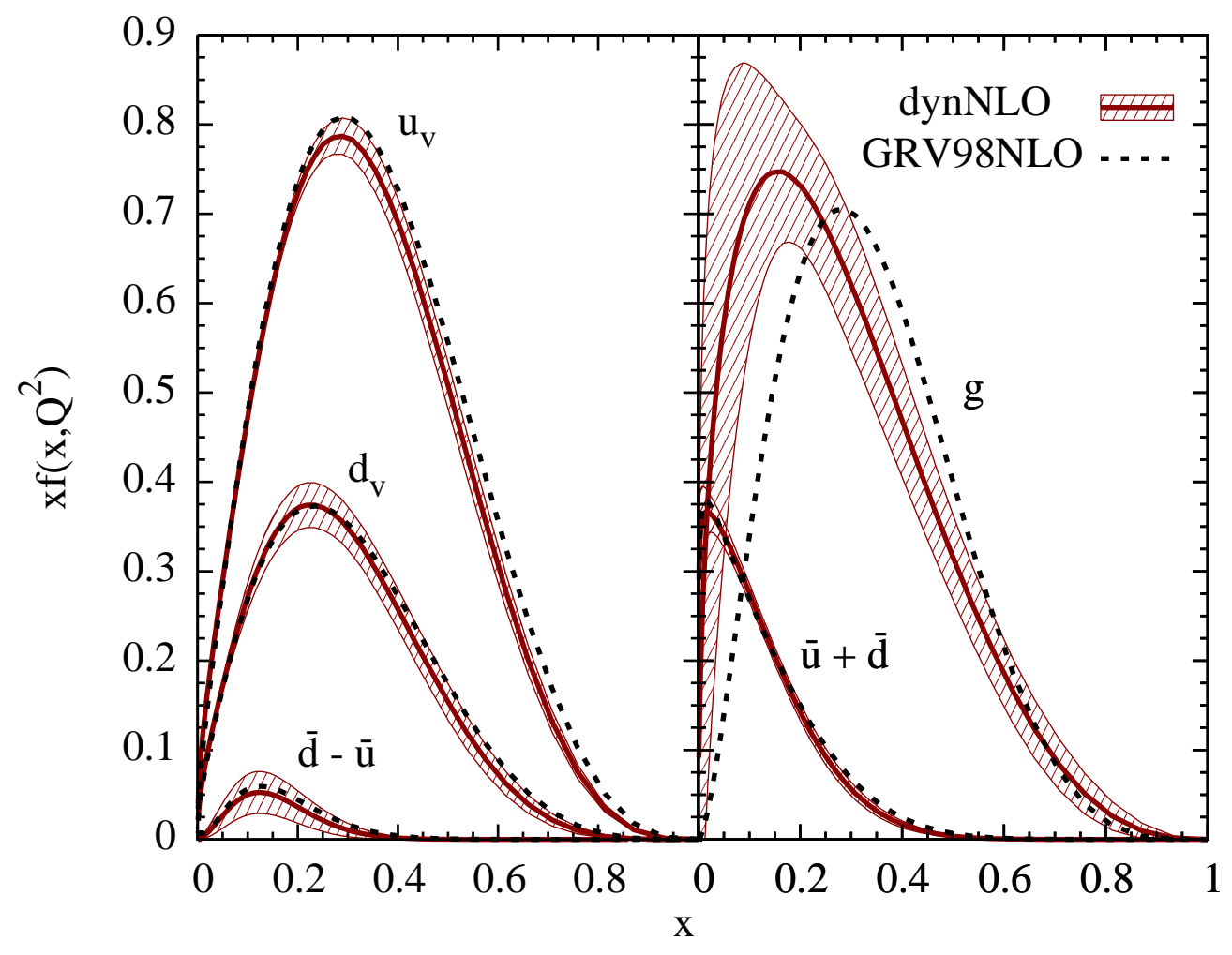

Figure 4: The valence-like input densities together with their $\pm 1 \sigma$ uncertainties at $Q_{0}^{2} \equiv$ $\mu_{\mathrm{NLO}}^{2}=0.5 \mathrm{GeV}^{2}$ for our dynamical $\mathrm{NLO}(\overline{\mathrm{MS}})$ results. The central curves follow from $(5)$ with the parameters given in Table 1 . The strange sea $s=\bar{s}$ vanishes at the input scale. The NLO GRV98 input [8] is also shown by the dashed curves for comparison. 

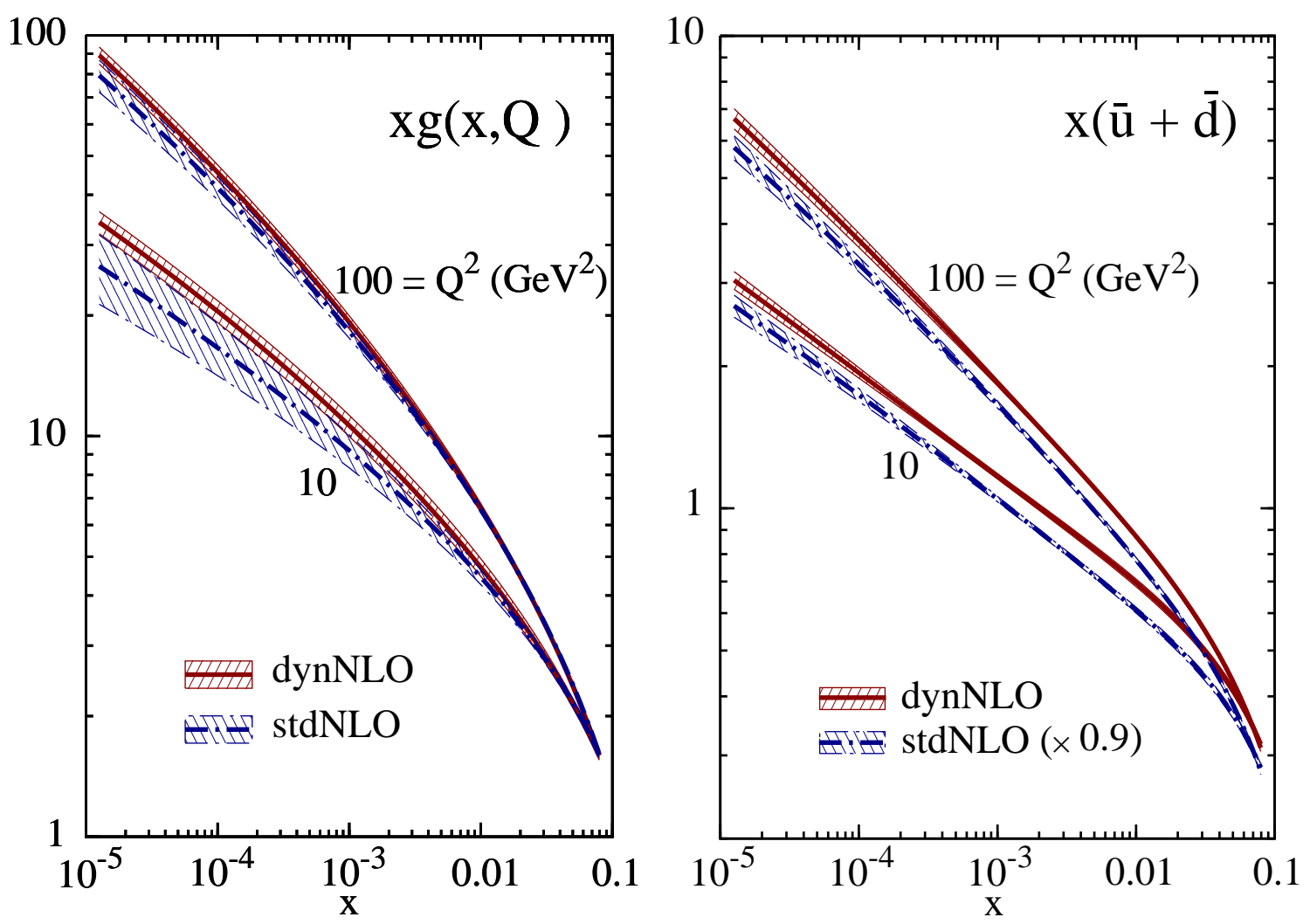

Figure 5: The small- $x$ NLO $(\overline{\mathrm{MS}})$ predictions of our dynamically (radiatively) generated gluon and sea-quark distributions, together with their $\pm 1 \sigma$ uncertainties, as compared to the results of a standard fit. To ease the visibility of the two error bands of $x(\bar{u}+\bar{d})$ we have multiplied the stdNLO results by 0.9 as indicated. The corresponding GRV98 predictions [8] lie within the $1 \sigma$ band of our new dynNLO results. 


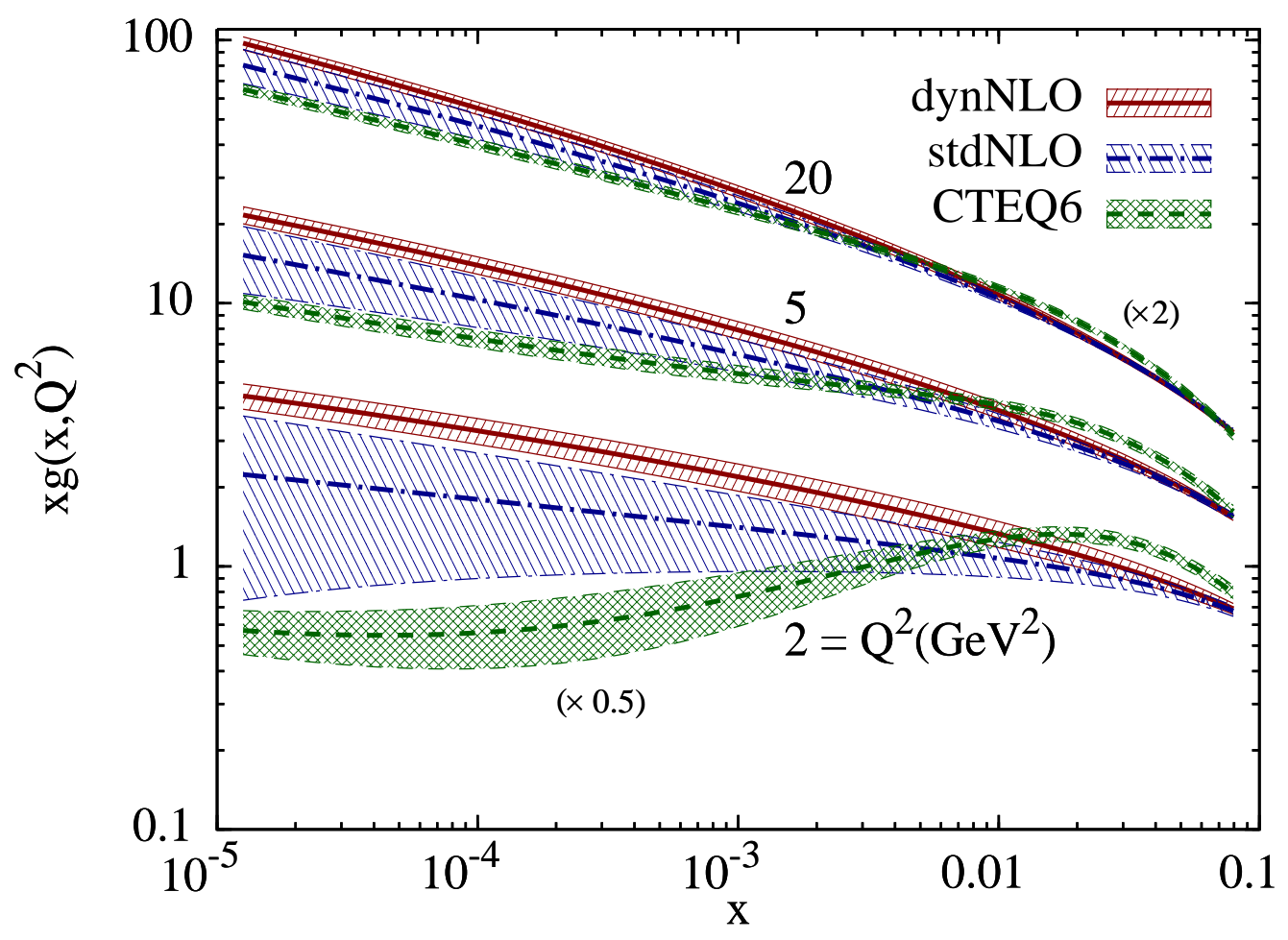

Figure 6: Comparing the $\pm 1 \sigma$ error bands of our dynamical (dyn), standard (std) and CTEQ [2] $\mathrm{NLO}(\overline{\mathrm{MS}})$ gluon distribution at small $x$ for various fixed values of $Q^{2}$. Note that $Q^{2}=2 \mathrm{GeV}^{2}$ is the input scale of the standard fit which is close to the CTEQ input scale $Q_{0}^{2}=m_{c}^{2} \simeq 1.7 \mathrm{GeV}^{2}$, where the standard CTEQ6 fit employs a valence-like gluon input (i.e., $x g\left(x, Q_{0}^{2}\right) \rightarrow 0$ as $x \rightarrow 0$ ). Due to the sizeably different input scales, the CTEQ6 gluon falls up to 30-40\% below our dynNLO gluon for $x<10^{-3}$ and $Q^{2}>10$ $\mathrm{GeV}^{2}$. The results at $Q^{2}=2$ and $20 \mathrm{GeV}^{2}$ have been multiplied by 0.5 and 2 , respectively, as indicated in the figure. 


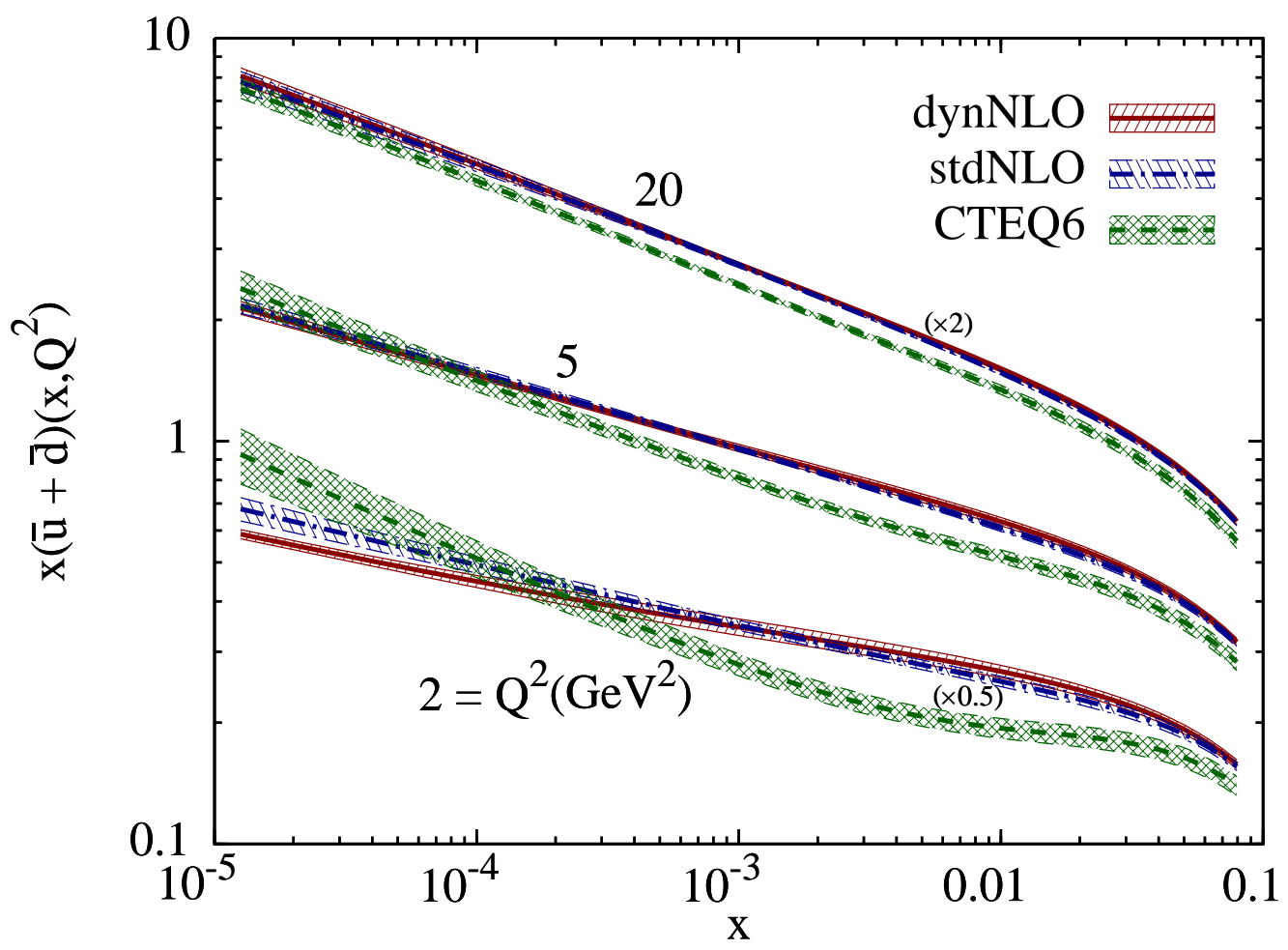

Figure 7: As in Fig. 6 but for the sea-quark distribution $x(\bar{u}+\bar{d})$. Notice that here the CTEQ input distribution at $Q_{0}^{2} \simeq 1.7 \mathrm{GeV}^{2}$ is not valence-like (i.e., $x(\bar{u}+\bar{d})\left(x, Q_{0}^{2}\right) \nrightarrow 0$ for $x \rightarrow 0$ ). 


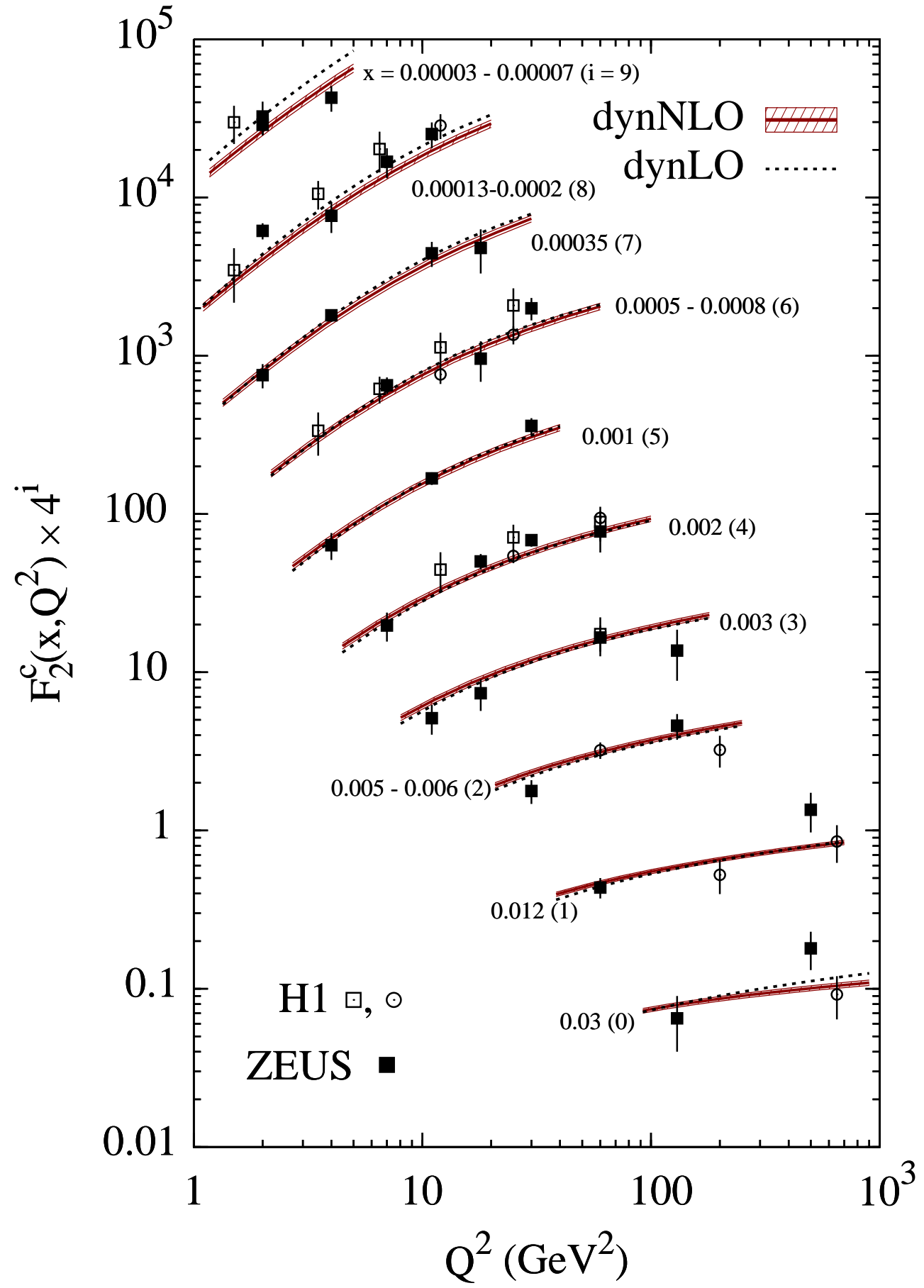

Figure 8: The dynamical NLO $(\overline{\mathrm{MS}})$ predictions for $F_{2}^{c}$ in the strict $n_{f}=3 \mathrm{FFNS}$, choosing $\mu_{F}^{2}=4 m_{c}^{2}$ with $m_{c}=1.3 \mathrm{GeV}$, together with the $\pm 1 \sigma$ uncertainty band. For comparison we also display the central LO predictions which are entirely due to the $\gamma^{*}$-gluon fusion subprocess $\gamma^{*} g \rightarrow c \bar{c}$. The charm production data as obtained from $D^{*}$ measurements are taken from [38, 39] (solid and open squares) and the H1 direct track measurements from [40] (open circles). 


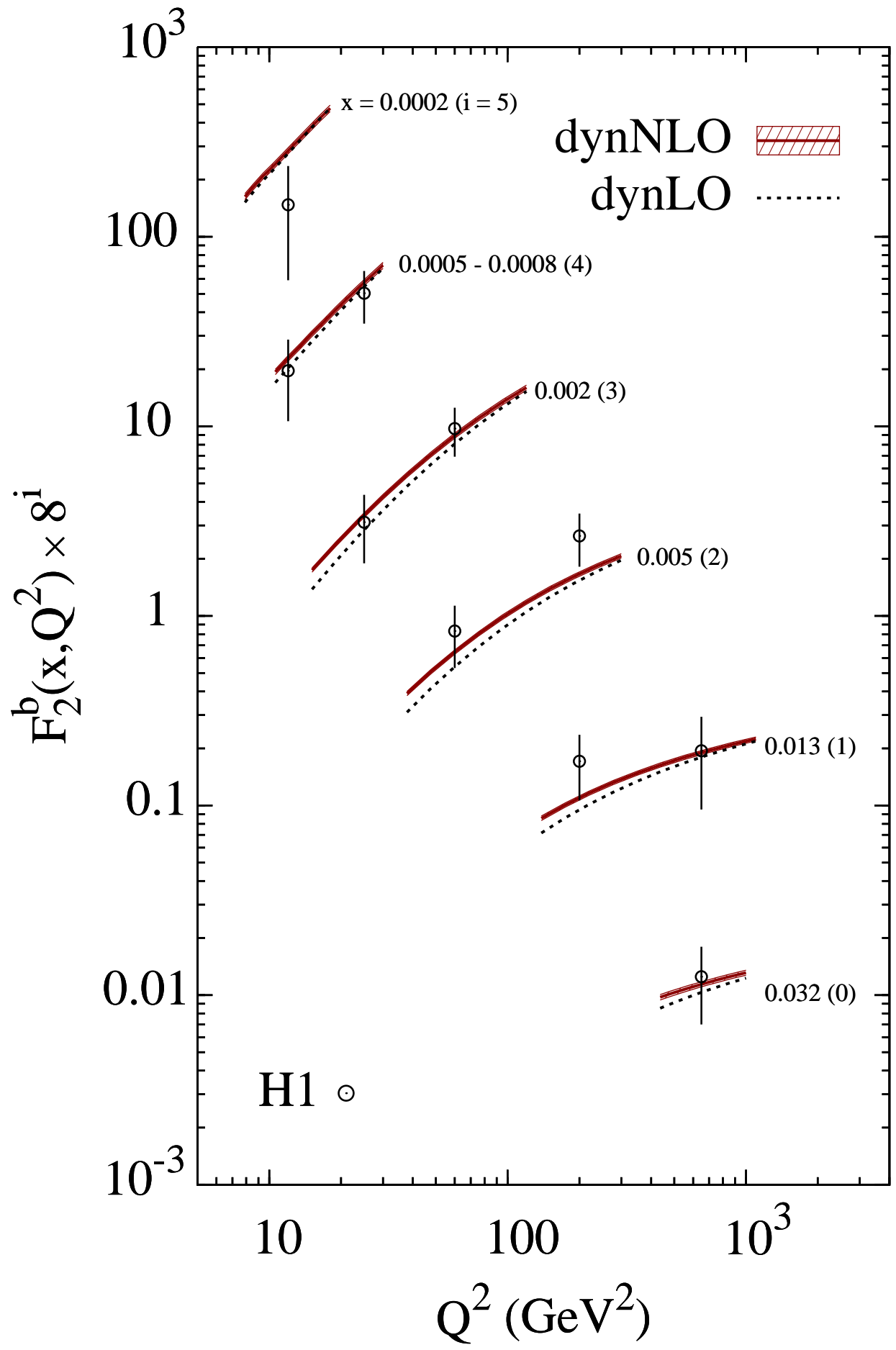

Figure 9: As in Fig. 8 but for $F_{2}^{b}$ with $m_{b}=4.2 \mathrm{GeV}$ and the bottom production data taken from [40]. 


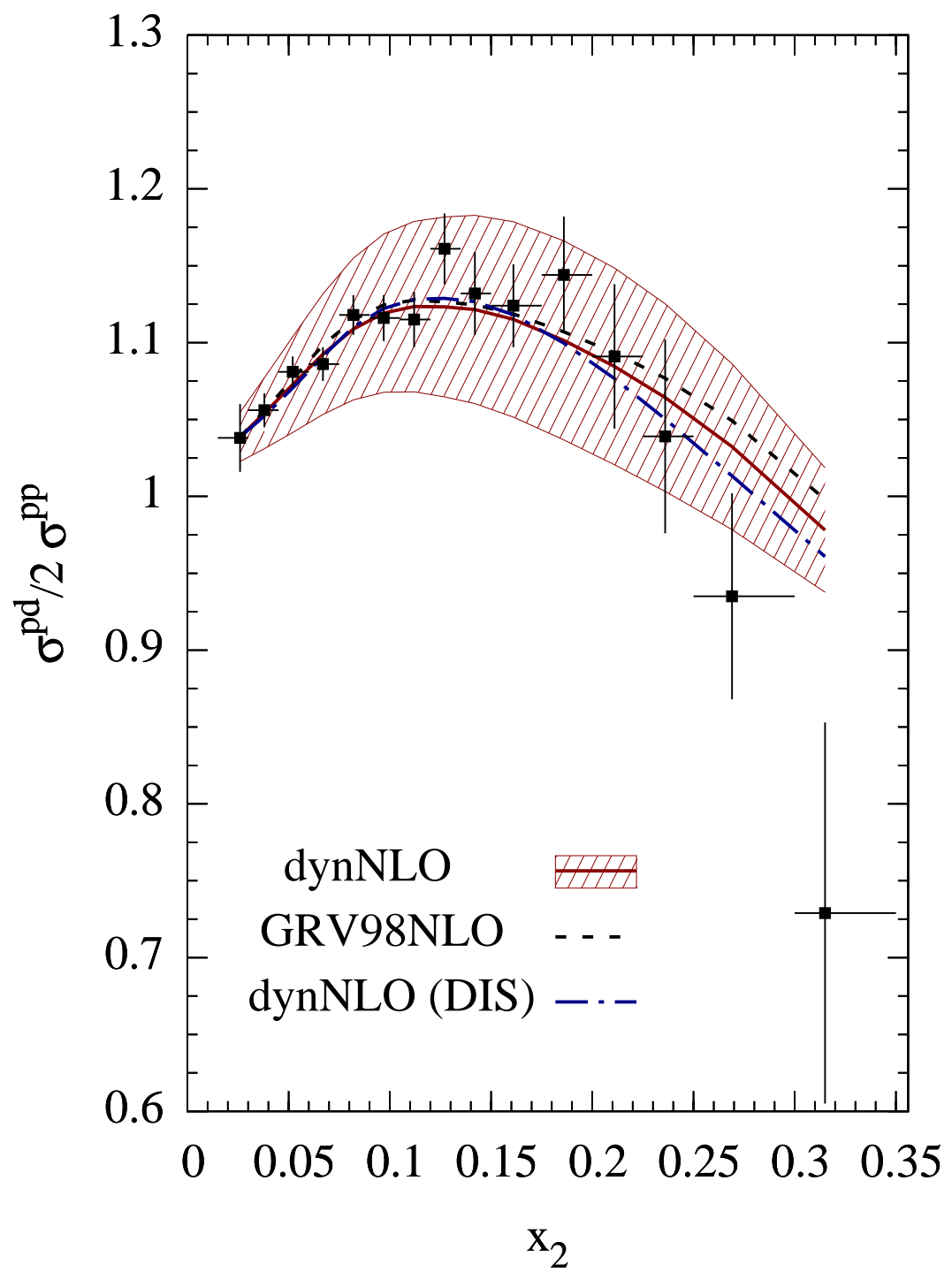

Figure 10: Our dynamical NLO result in the $\overline{\mathrm{MS}}$ factorization scheme, together with its $\pm 1 \sigma$ uncertainty, for $\sigma^{p d} / 2 \sigma^{p p}$ appearing in the Drell-Yan asymmetry $A_{D Y}=\left(\sigma^{p p}-\right.$ $\left.\sigma^{p n}\right) /\left(\sigma^{p p}+\sigma^{p n}\right)$ as a function of the average fractional momentum $x_{2}$ of the target partons. The GRV98 NLO MS-result [8] is shown for comparison. The dynamical NLO(DIS) result in the DIS factorization scheme is shown by the dashed-dotted curve. The data for the dimuon mass range $4.6 \leq M_{\mu^{+} \mu^{-}} \leq 12.9 \mathrm{GeV}$ are taken from [42]. 


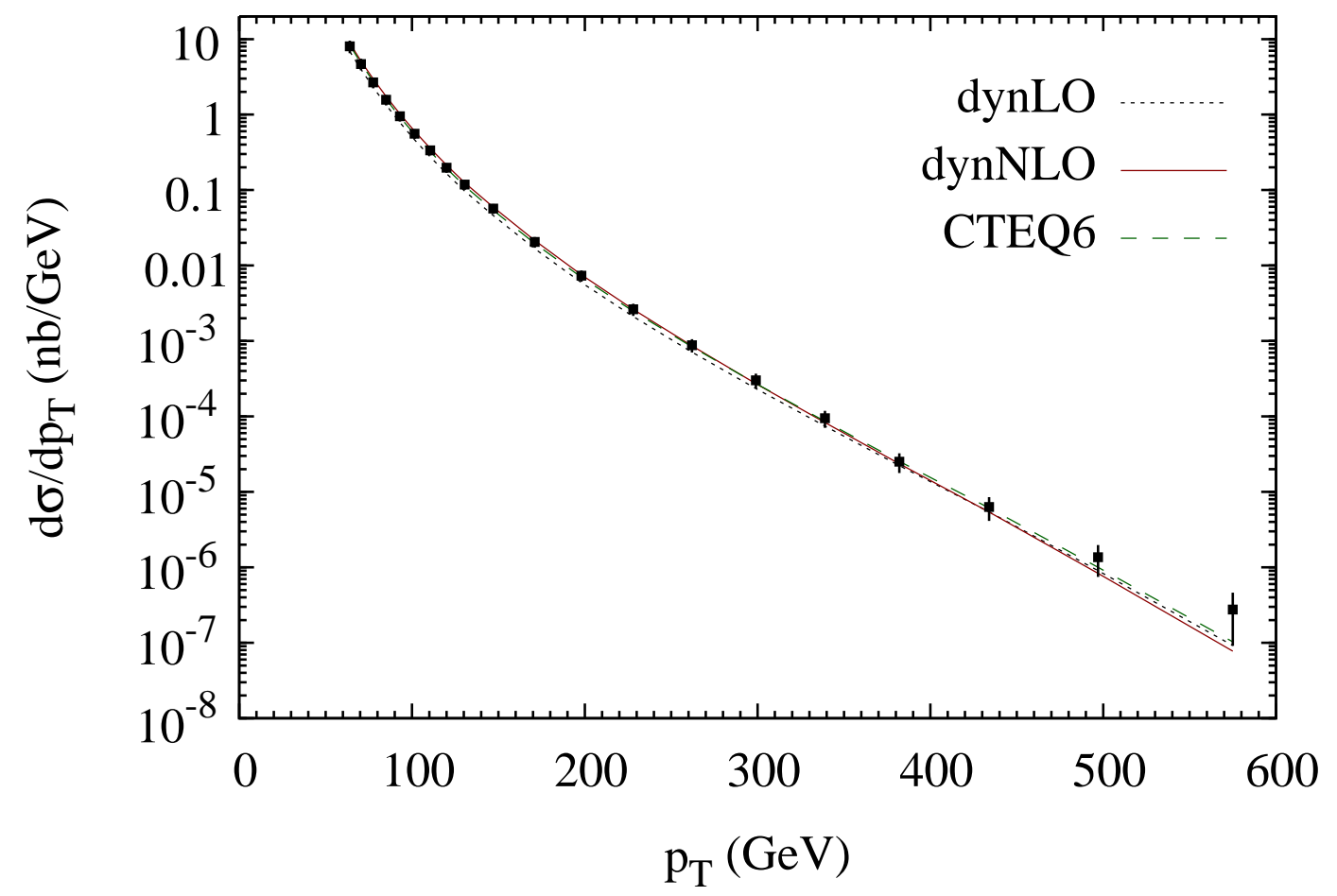

Figure 11: The $p \bar{p}$ Tevatron high $-p_{\mathrm{T}}$ inclusive jet data [46, 47] compared with our dynamical LO and $\mathrm{NLO}(\overline{\mathrm{MS}})$ results, as well as with the NLO CTEQ6 result [2]. 


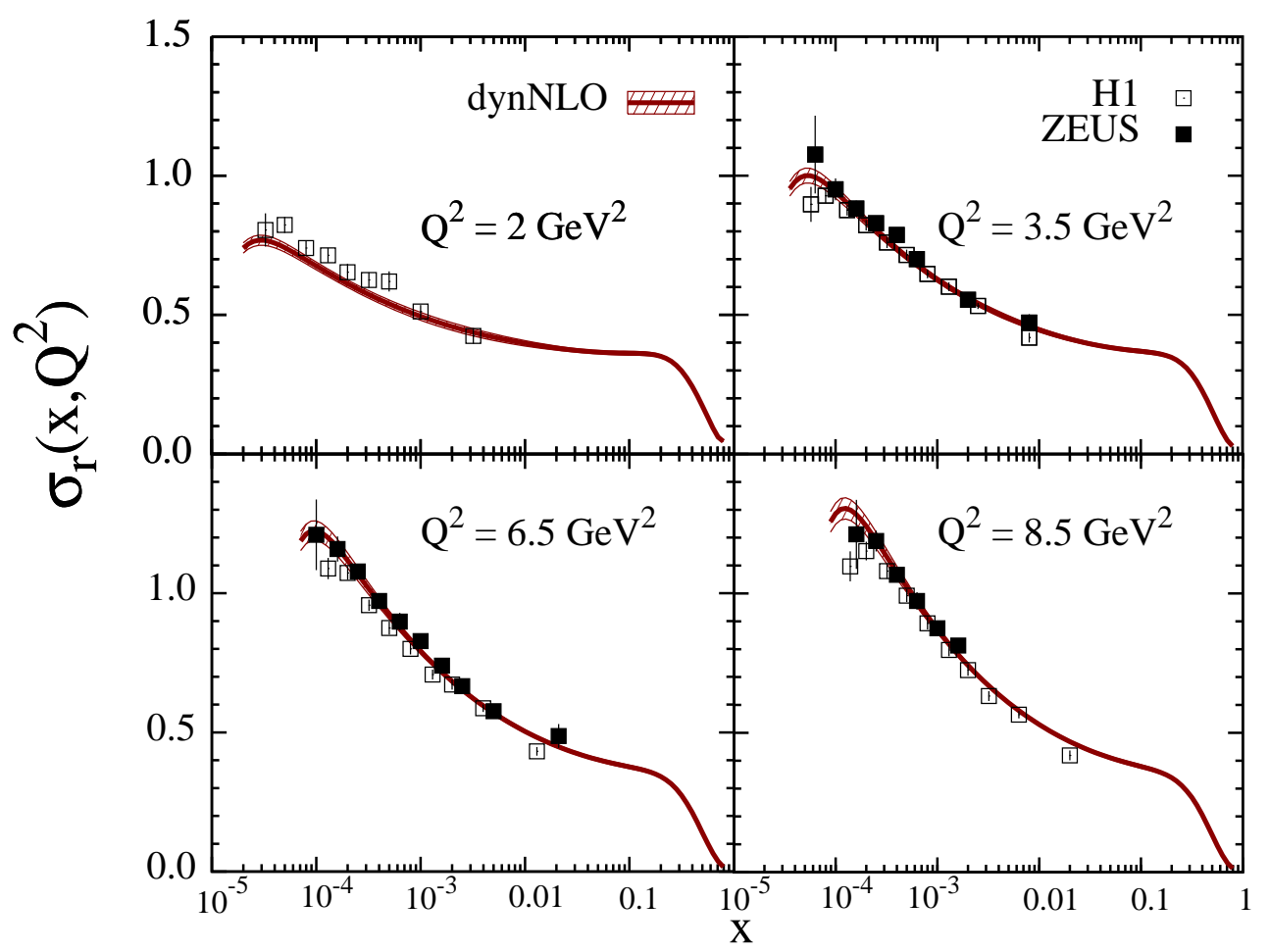

Figure 12: The dynamical NLO $(\overline{\mathrm{MS}})$ predictions, together with their $\pm 1 \sigma$ uncertainties, for the 'reduced' DIS cross section $\sigma_{r}\left(x, Q^{2}\right)=F_{2}-\left(y^{2} / Y_{+}\right) F_{\mathrm{L}}$. The HERA data for some representative fixed values of $Q^{2}$ are from [28, 29]. 


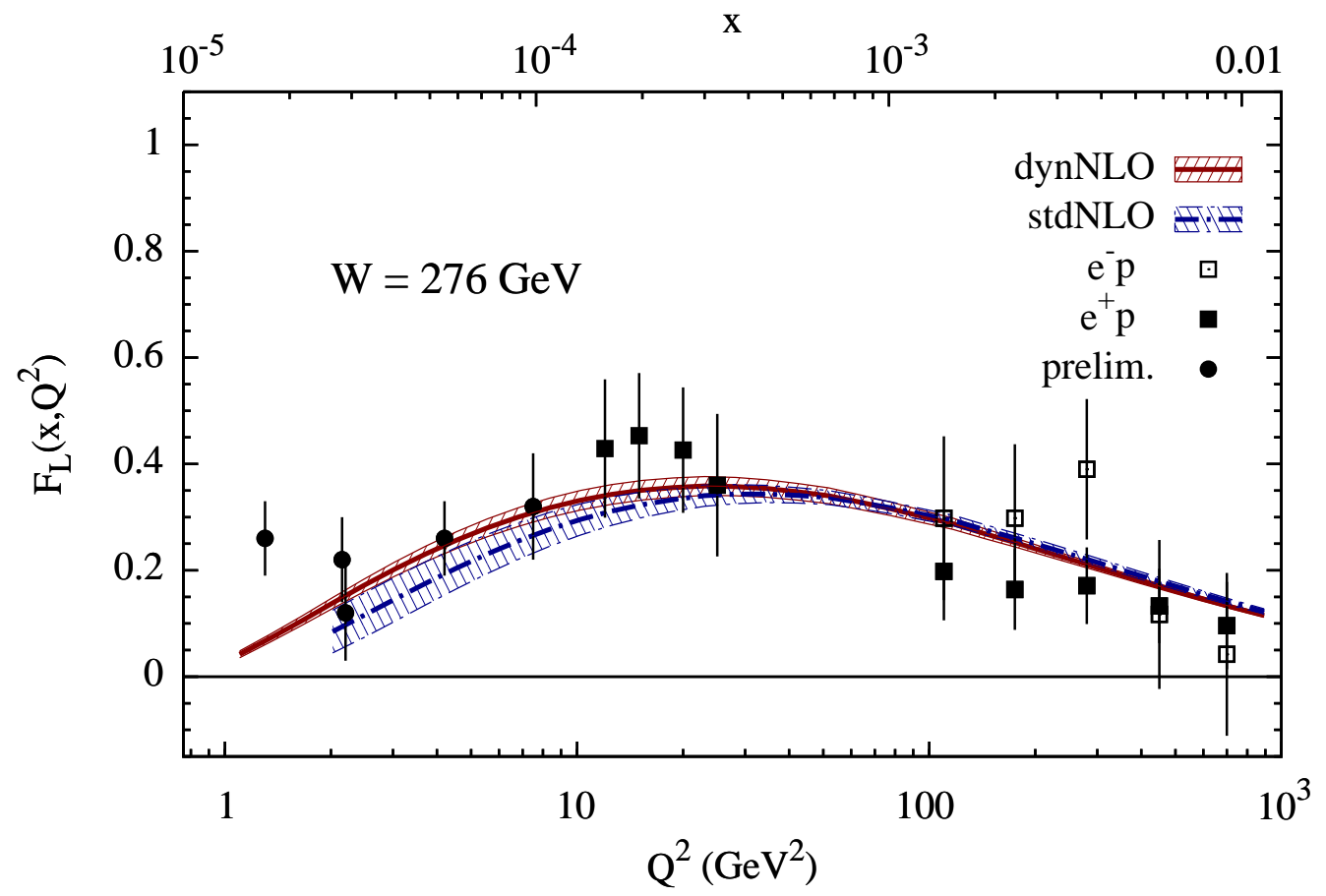

Figure 13: Dynamical and standard $\operatorname{NLO}(\overline{\mathrm{MS}})$ results for $F_{\mathrm{L}}\left(x, Q^{2}\right)$ together with their $\pm 1 \sigma$ uncertainty bands. The (partly preliminary) $\mathrm{H} 1$ data [28, 64] are at fixed $W \simeq 276$ $\mathrm{GeV}$. 


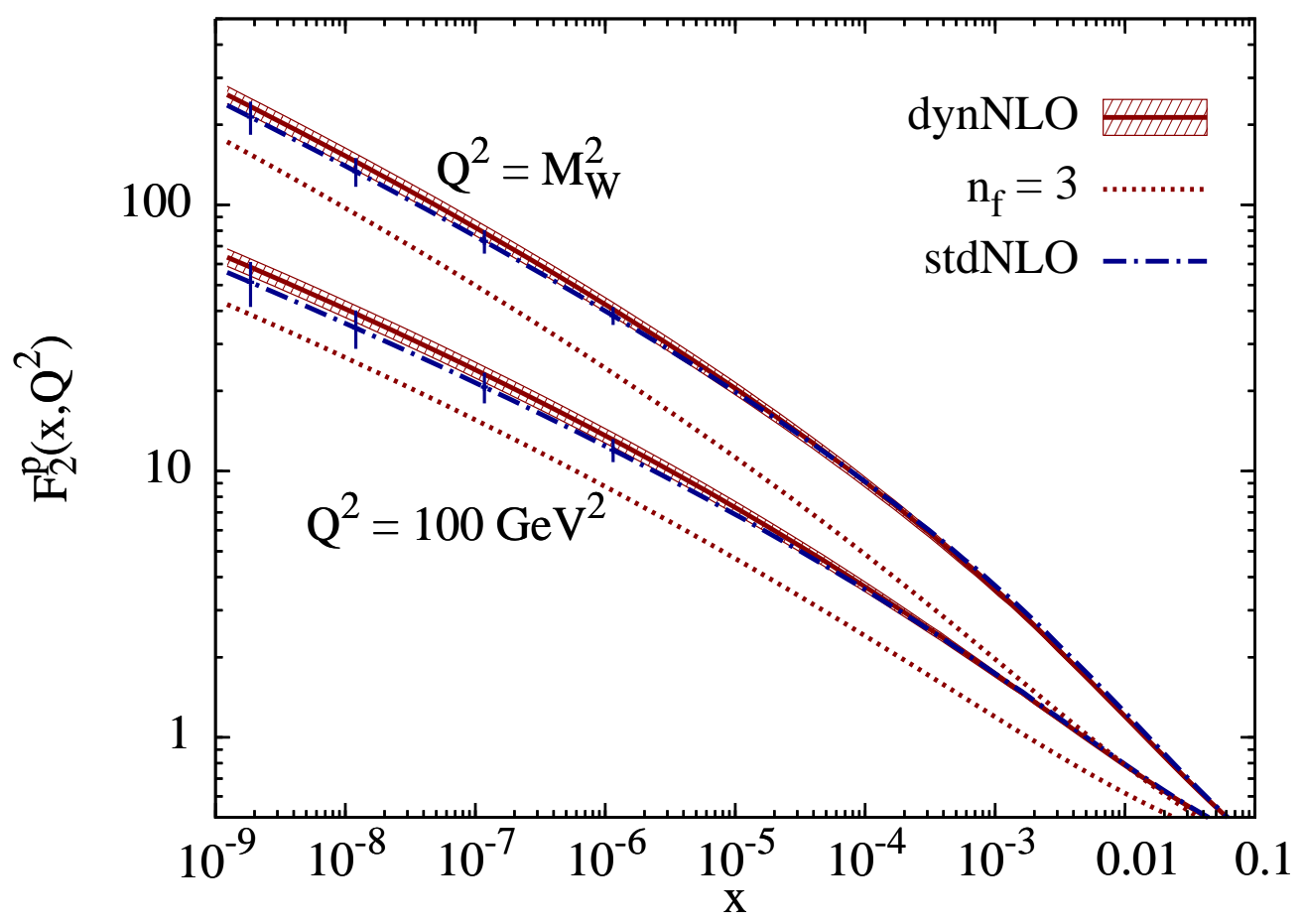

Figure 14: Dynamical NLO $(\overline{\mathrm{MS}})$ predictions for $F_{2}^{p}\left(x, Q^{2}\right)$, together with their $1 \sigma$ uncertainties, for extremely small values of $x$. The $1 \sigma$ uncertainties of the standard (std) NLO fit extrapolations are shown by the vertical bars. The dotted curves are the contributions from the light $\left(n_{f}=3\right)$ quarks and gluons to $F_{2}^{p}$ for the dynamical (dyn) NLO result. In other words, the difference between the dotted and solid curves is due to NLO heavy quark (charm, bottom) contributions which derive from photon-gluon (quark) fusion processes. The dynamical GRV98 predictions [8] lie within the $\pm 1 \sigma$ band of our present dynNLO predictions. 

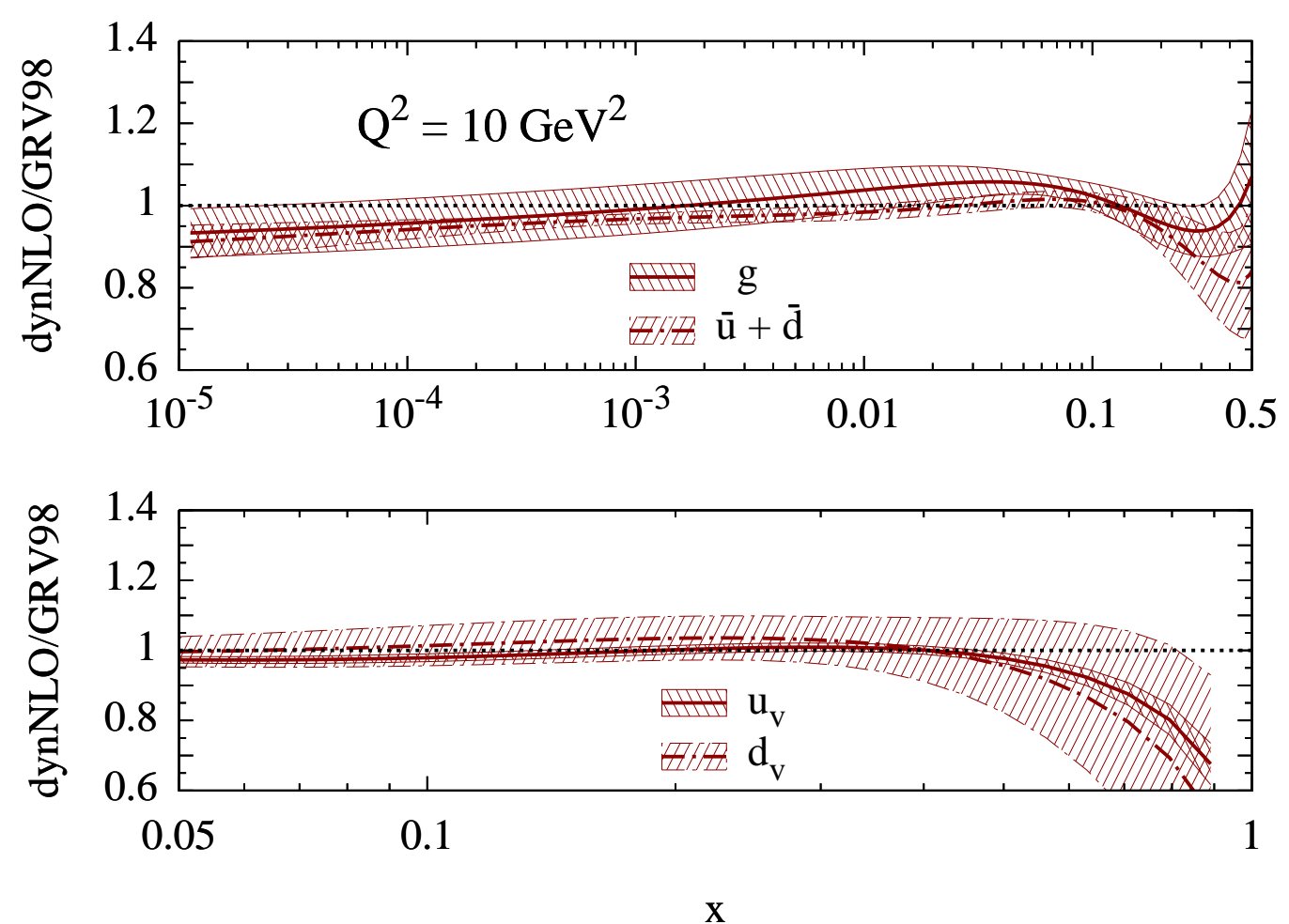

Figure 15: Comparing the present dynamical dynNLO $(\overline{\mathrm{MS}})$ parton distributions with the previous ones of GRV98 [8] at $Q^{2}=10 \mathrm{GeV}^{2}$. 


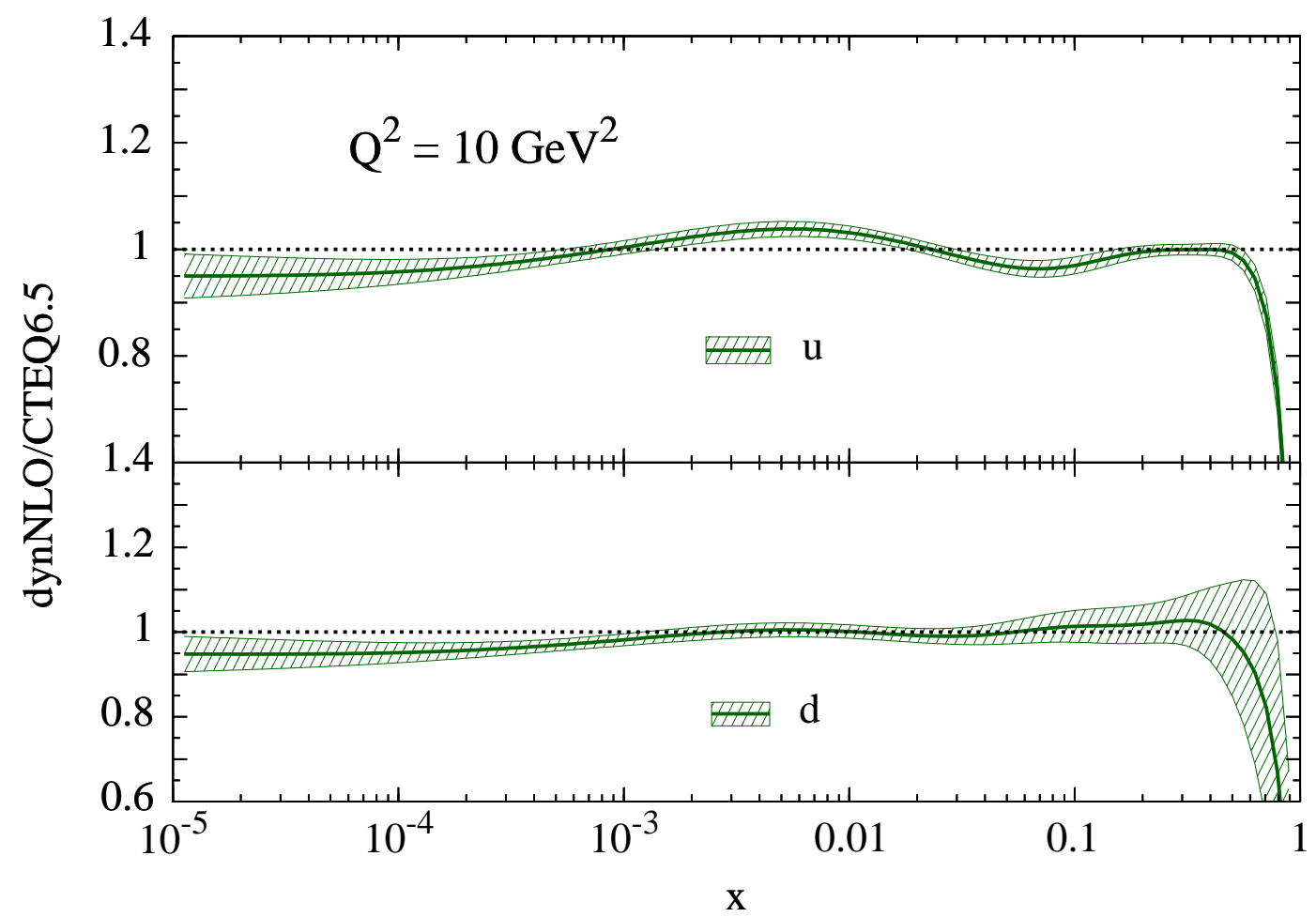

Figure 16: Comparing the present dynamical $\operatorname{NLO}(\overline{\mathrm{MS}}) u=u_{v}+\bar{u}$ and $d=d_{v}+\bar{d}$ distributions with the ones of CTEQ6.5 [70] at $Q^{2}=10 \mathrm{GeV}^{2}$. These ratios remain practically unchanged at higher scales, like $Q^{2}=M_{W}^{2}$ relevant for $W^{ \pm}$production. The shaded areas represent the estimated $\pm 1 \sigma$ uncertainty band of our dynNLO analysis. Notice that in the relevant small- $x$ region these ratios would be practically unaltered if the GRV98 distributions [8] were used instead of the dynNLO ones, since dynNLO/GRV98 $\simeq 1$ as evident from Fig. 15 . 